\title{
THETA FUNCTIONS ON HERMITIAN SYMMETRIC DOMAINS AND FOCK REPRESENTATIONS
}

\author{
MIN HO LEE \\ (Received 30 January 2001; revised 8 November 2001) \\ Communicated by A. H. Dooley
}

\begin{abstract}
One way of realizing representations of the Heisenberg group is by using Fock representations, whose representation spaces are Hilbert spaces of functions on complex vector space with inner products associated to points on a Siegel upper half space. We generalize such Fock representations using inner products associated to points on a Hermitian symmetric domain that is mapped into a Siegel upper half space by an equivariant holomorphic map. The representations of the Heisenberg group are then given by an automorphy factor associated to a Kuga fiber variety. We introduce theta functions associated to an equivariant holomorphic map and study connections between such generalized theta functions and Fock representations described above. Furthermore, we discuss Jacobi forms on Hermitian symmetric domains in connection with twisted torus bundles over symmetric spaces.
\end{abstract}

2000 Mathematics subject classification: primary 22E45, 11F55, 11F27; secondary 14K 10, 14K25.

\section{Introduction}

Let $\mathscr{H}_{n}$ be the Siegel upper half space of degree $n$ consisting of complex symmetric $n \times n$ matrices with positive imaginary part. Then the symplectic group $\mathrm{Sp}(n, \mathbb{R})$ acts on $\mathscr{H}_{n}$, and the quotient $\Gamma^{\prime} \backslash \mathscr{H}_{n}$ of $\mathscr{H}_{n}$ by an arithmetic subgroup $\Gamma^{\prime}$ of $\mathrm{Sp}(n, \mathbb{R})$ can be regarded as the moduli space for a certain family of polarized abelian varieties, known as a universal family (see for example $[5,10,13]$ ). Such a family of abelian varieties can be considered as a fiber variety over the Siegel modular variety $X^{\prime}=\Gamma^{\prime} \backslash \mathscr{H}_{n}$, and the geometry of a Siegel modular variety and the associated universal family of abelian varieties are closely connected with various topics in number theory including the theory of Siegel modular forms, theta functions and Jacobi forms.

(C) 2003 Australian Mathematical Society $1446-7887 / 03 \$ A 2.00+0.00$ 
Let $G=\mathbb{G}(\mathbb{R})$ be a semisimple Lie group of Hermitian type that can be realized as the set of real points of a linear algebraic group $\mathbb{G}$ defined over $\mathbb{Q}$. Thus the quotient $\mathscr{D}=G / K$ of $G$ by a maximal compact subgroup $K$ has the structure of a Hermitian symmetric domain. Let $\tau: \mathscr{D} \rightarrow \mathscr{H}_{n}$ be a holomorphic map, and let $\rho: G \rightarrow \operatorname{Sp}(n, \mathbb{R})$ be a homomorphism of $G$ into a real symplectic group of degree $n$ such that $\tau(g z)=\rho(g) \tau(z)$ for all $z \in \mathscr{D}$ and $g \in G$. Let $\Gamma$ be an arithmetic subgroup of $G$ such that $\rho(\Gamma) \subset \Gamma^{\prime}$, and let $X=\Gamma \backslash \mathscr{D}$ be the associated arithmetic variety. Then $\tau$ induces a morphism $\tau_{X}: X \rightarrow X^{\prime}$ of arithmetic varieties, and by pulling back the fiber variety over $X^{\prime}$ via $\tau_{X}$ we obtain a fiber variety over $X$ whose fibers are again polarized abelian varieties (see Section 2 for details). Such fiber varieties over an arithmetic variety are called Kuga fiber varieties (see [12, 22]), and various geometric and arithmetic aspects of Kuga fiber varieties have been investigated in numerous papers (see for example $[1,2,8,14,16]$ ). Various objects connected with Siegel modular varieties and the associated universal families of abelian varieties can be generalized to the corresponding objects connected with more general locally symmetric varieties and the associated Kuga fiber varieties.

One of the important nilpotent Lie groups is the Heisenberg group whose irreducible representations were classified by Stone and von Neumann (see for example $[11,19,23])$. One way of realizing representations of the Heisenberg group is by using Fock representations, whose representation spaces are Hilbert spaces of functions on complex vector spaces with inner products associated to points on a Siegel upper half space (see [21]). In this paper, we generalize such Fock representations using inner products associated to points on a Hermitian symmetric domain that is mapped into a Siegel upper half space by an equivariant holomorphic map. The representations of the Heisenberg group are then given by an automorphy factor associated to a Kuga fiber variety. We introduce theta functions associated to an equivariant holomorphic map and study connections between such generalized theta functions and Fock representations described above. Furthermore, we discuss Jacobi forms on Hermitian symmetric domains in connection with twisted torus bundles over locally symmetric spaces (see [15]).

This paper is organized as follows. In Section 2, we describe the construction of Kuga fiber varieties over an arithmetic variety. In Section 3 we review the notion of canonical automorphy factors for groups of Hermitian type, and automorphy factors associated to products of semisimple groups and Heisenberg groups are discussed in Section 4. In Section 5, we construct twisted torus bundles over arithmetic varieties which are circle bundles over Kuga fiber varieties. We then study their connections with Jacobi forms on Hermitian symmetric domains. Section 6 is devoted to the discussion of Fock representations of Heisenberg groups on the space $\mathscr{F}_{z}$ of certain functions on vector spaces associated to each $z \in \mathscr{D}$. We prove that such representations are unitary and irreducible. In Section 7 we introduce theta functions on 
Hermitian symmetric domains and prove that certain types of such theta functions generate the eigenspace of the Fock representation associated to a quasi-character.

\section{Kuga fiber varieties}

In this section we review the construction of Kuga fiber varieties associated to equivariant holomorphic maps of symmetric domains. They are fiber bundles over locally symmetric spaces whose fibers are polarized abelian varieties. More details can be found in [12] and [22].

Let $G$ be a Zariski-connected semisimple real algebraic group of Hermitian type defined over $\mathbb{Q}$. Thus $G$ is the set of real points $\mathbb{G}(\mathbb{R})$ of a semisimple algebraic group defined over $\mathbb{Q}$, and the associated Riemannian symmetric space $\mathscr{D}=G / K$, where $K$ is a maximal compact subgroup, has a $G$-invariant complex structure. Such a space can be identified with a bounded symmetric domain in $\mathbb{C}^{k}$ for some $k$, and is called a Hermitian symmetric domain. Let $G^{\prime}$ be another group of the same type, and let $\mathscr{D}^{\prime}$ be the associated Hermitian symmetric domain. A pair $(\rho, \tau)$ consisting of a homomorphism $\rho: G \rightarrow G^{\prime}$ and a holomorphic map $\tau: \mathscr{D} \rightarrow \mathscr{D}^{\prime}$ is said to be equivariant if $\tau(g z)=\rho(g) \tau(z)$ for all $g \in G$ and $z \in \mathscr{D}$.

Let $V$ be a real vector space of dimension $2 n$ defined over $\mathbb{Q}$, and let $\beta$ be a nondegenerate alternating bilinear form on $V$ defined over $\mathbb{Q}$. Then the symplectic group

$$
\mathrm{Sp}(V, \beta)=\left\{g \in \mathrm{GL}(V) \mid \beta\left(g v, g v^{\prime}\right)=\beta\left(v, v^{\prime}\right) \text { for all } v, v^{\prime} \in V\right\}
$$

is of Hermitian type, and the associated Hermitian symmetric domain can be identified with the set $\mathscr{H}=\mathscr{H}(V, \beta)$ of all complex structures $I$ on $V$ such that the bilinear form $V \times V \rightarrow \mathbb{R},\left(v, v^{\prime}\right) \mapsto \beta\left(v, I v^{\prime}\right)$ is symmetric and positive definite. The action of $\operatorname{Sp}(V, \beta)$ on $\mathscr{H}$ is given by the map $G \times \mathscr{H} \rightarrow \mathscr{H},(g, l) \mapsto g I g^{-1}$. Let $\left\{e_{1}, \ldots, e_{2 n}\right\}$ be a symplectic basis of $(V, \beta)$, that is, a basis of $V$ satisfying the condition

$$
\beta\left(e_{i}, e_{j}\right)= \begin{cases}1 & \text { if } i=j+n \\ -1 & \text { if } i=j-n \\ 0 & \text { otherwise }\end{cases}
$$

for $1 \leq i, j \leq 2 n$. Then $\operatorname{Sp}(V, \beta)$ can be identified with the real symplectic group $\operatorname{Sp}(n, \mathbb{R})$ of degree $n$, and $\mathscr{H}$ can be identified with the Siegel upper half space

$$
\mathscr{H}_{n}=\left\{Z \in M_{n}(\mathbb{C}) \mid{ }^{t} Z=Z, \quad \operatorname{Im} Z \gg 0\right\}
$$


of degree $n$ on which $\operatorname{Sp}(n, \mathbb{R})$ acts as usual by

$$
g(Z)=(A Z+B)(C Z+D)^{-1} \text { for } g=\left(\begin{array}{ll}
A & B \\
C & D
\end{array}\right) \in \operatorname{Sp}(n, \mathbb{R})
$$

and $Z \in \mathscr{H}_{n}$.

We now consider an equivariant pair $(\rho, \tau)$ for the special case of $G^{\prime}=\operatorname{Sp}(V, \beta)$. Thus the homomorphism $\rho: G \rightarrow \operatorname{Sp}(V, \beta)$ and the holomorphic map $\tau: \mathscr{D} \rightarrow \mathscr{H}_{n}$ satisfies the condition $\tau(g z)=\rho(g) \tau(z)$ for all $g \in G$ and $z \in \mathscr{D}$. Let $\Gamma$ be a torsionfree arithmetic subgroup of $G$, and let $L$ be a lattice in $V$ with $V_{\mathbf{Q}}=L \otimes_{\mathbf{Z}} \mathbb{Q}$ such that $\beta(L, L) \subset \mathbb{Z}$ and $\rho(\Gamma) L \subset L$. Regarding $\mathscr{H}_{n}$ as the set of complex structures on $V$, each element $z \in \mathscr{D}$ determines a complex vector space $\left(V, I_{\tau(z)}\right)$, where $I_{\tau(z)}$ is the complex structure on $V$ corresponding to $\tau(z) \in \mathscr{H}_{n}$. Let $z_{0}$ be a fixed element of $\mathscr{D}$, and let $I_{0}$ be the complex structure on $V$ corresponding to the element $\tau\left(z_{0}\right)$ of $\mathscr{H}_{n}$. Let $V_{\mathrm{C}}=V \otimes_{\mathrm{R}} \mathbb{C}$ be the complexification of $V$, and denote by $V_{+}$and $V_{-}$the subspaces of $V_{\mathrm{C}}$ defined by $V_{ \pm}=\left\{v \in V_{\mathrm{C}} \mid I_{0} v= \pm i v\right\}$, so that we have

$$
V_{\mathrm{c}}=V_{+} \oplus V_{-}, \quad V_{+}=\bar{V}_{-} .
$$

Then each element $v$ in $\left(V, I_{\tau(z)}\right)$ determines an element

$$
\xi(z, v)=v_{z}=v_{+}-\tau(z) v_{-}=v_{+}-I_{\tau(z)} v_{-}
$$

of the subspace $V_{+}$of $V_{\mathrm{C}}$, where the elements $v_{ \pm}$denote the $V_{ \pm}$-components of $v \in V \subset V_{\mathrm{C}}=V_{+} \oplus V_{-}$. We set

$$
W=\coprod_{z \in \mathscr{D}}\left(V, I_{\tau(z)}\right),
$$

the disjoint union of the vector spaces $\left(V, I_{\tau(z)}\right)$ with complex structure $I_{\tau(z)}$ for the elements $z \in \mathscr{D}$. Then the map

$$
W \rightarrow \mathscr{D} \times V_{+}, \quad(z, v) \mapsto(z, \xi(z, v))
$$

determines a bijection $W \cong \mathscr{D} \times V_{+}$and a $\mathbb{C}$-linear isomorphism $\left(V, I_{\tau(z)}\right) \cong\{z\} \times V_{+}$. Thus the natural projection map $W \rightarrow \mathscr{D}$ has the structure of a holomorphic vector bundle with fiber $V_{+}$.

Let $G \ltimes_{\rho} V$ be the semidirect product of $G$ and $V$ with respect to the action of $G$ on $V$ via $\rho$. Thus $G \ltimes_{\rho} V$ consists of the elements $(g, v) \in G \times V$ and its multiplication operation is given by $(g, v)\left(g^{\prime}, v^{\prime}\right)=\left(g g^{\prime}, \rho(g) v^{\prime}+v\right)$ for $g, g^{\prime} \in G$ and $v, v^{\prime} \in V$. Then $G \ltimes_{\rho} V$ acts on $W$ by

$$
(g, v)(z, w)=(g z, \rho(g) w+\rho(g) v)
$$

for $(g, v) \in G \ltimes_{\rho} V, z \in \mathscr{D}$ and $w \in\left(V, I_{\mathbf{r}(z)}\right)$. 
REMARK 2.1. Let $g$ be a fixed element of $G$. Then we have

$$
I_{\tau(g z)}=I_{\rho(g) \tau(z)}=\rho(g) I_{\tau(z)} \rho(g)^{-1}
$$

for all $z \in \mathscr{D}$. Consider the map $\phi_{g}: V \rightarrow V$ defined by $\phi_{g}(v)=\rho(g) v$ for all $v \in V$. Then $\phi_{g}$ is an $\mathbb{R}$-linear isomorphism of $V$ such that $I_{\tau(g z)} \circ \phi_{g}=\phi_{g} \circ I_{\tau(z)}$ for all $z \in \mathscr{D}$, that is, $\phi_{g}$ is an isomorphism between the vector spaces with complex structure $\left(V, I_{\tau(z)}\right)$ and $\left(V, I_{\tau(g \tau)}\right)$. Indeed, we have

$$
\left(I_{\tau(g z)} \circ \phi_{g}\right)(v)=\rho(g) I_{\tau(z)} \rho(g)^{-1} \rho(g) v=\rho(g) I_{\tau(z)} v=\left(\phi_{g} \circ I_{\tau(z)}\right)(v)
$$

for all $z \in \mathscr{D}$ and $v \in V$. Thus $\rho(g) v$ in (2.3) is simply the element of $\left(V, I_{\tau(g z)}\right)$ corresponding to the element $v$ in $\left(V, I_{\mathrm{r}(z)}\right)$.

Using the isomorphism between $W$ and $\mathscr{D} \times V_{+}$given in (2.2), wee see that, if $w=\xi(z, u)$ with $u \in\left(V, l_{\tau(z)}\right), G \ltimes_{\rho} V$ acts on $\mathscr{D} \times V_{+}$by

$$
\begin{aligned}
(g, v)(z, w) & =(g z, \xi(g z, \rho(g) u)+\xi(g z, \rho(g) v)) \\
& =\left(g z, \xi(g z, \rho(g) u)+(\rho(g) v)_{g z}\right)
\end{aligned}
$$

for $(g, v) \in G \ltimes_{\rho} V$ and $(z, w) \in \mathscr{D} \times V_{+}$. Since $\rho(g) v$ is the element of $\left(V, I_{\tau(g z)}\right)$ corresponding to $v \in\left(V, I_{\tau(z)}\right)$ as was mentioned in Remark 2.1, we shall simply write $v_{g z}$ for $(\rho(g) v)_{g z}$. Thus we have

$$
(g, v)(z, w)=\left(g z, \xi(g z, \rho(g) u)+v_{g z}\right)
$$

for all $(g, v) \in G \ltimes_{\rho} V$ and $(z, w) \in \mathscr{D} \times V_{+}$, where $u$ is an element of $\left(V, I_{\tau(z)}\right)$ such that $w=\xi(z, u)$.

Let the arithmetic subgroup $\Gamma \subset G$ and the lattice $L \subset V$ be as before. Then, since $\rho(\Gamma) L \subset L$, the action of $G \ltimes_{\rho} V$ on $W$ induces an action of the discrete subgroup $\Gamma \ltimes_{\rho} L$ of $G \ltimes_{\rho} V$ on $W$. Since $\Gamma$ is assumed to be torsion-free, the quotient spaces $X=\Gamma \backslash \mathscr{D}$ and $Y=\Gamma \ltimes_{\rho} L \backslash W$ are complex manifolds. The natural projection map $W \rightarrow \mathscr{D}$ determines the structure of a fiber bundle on the induced projection map $\pi: Y \rightarrow X$ whose fiber over $\Gamma z \in X$ with $z \in \mathscr{D}$ is the quotient $\left(V, I_{\tau(z)}\right) / L$ of the complex vector space $\left(V, I_{\tau(z)}\right)$ by the lattice $L$. The complex torus $\left(V, I_{\tau(z)}\right) / L$ is in fact an abelian variety because the alternating bilinear form $\beta$ can be used as a Riemann form. Thus we obtain a fiber bundle $\pi: Y \rightarrow X$ whose fibers are abelian varieties of the form $\left(V, I_{r(z)}\right) / L$. The total space $Y$ of such a fiber bundle is called a Kuga fiber variety. 


\section{Canonical automorphy factors}

In this section we review the notion of canonical automorphy factors for semisimple Lie groups of Hermitian type and describe some of their properties (see [20, 22]). Let $G=\mathbb{G}(\mathbb{R})$ be a semisimple real algebraic group of Hermitian type as in Section 2, and let $K$ be a maximal compact subgroup of $G$. Then the Riemannian symmetric space $\mathscr{D}=G / K$ has a $G$-invariant complex structure $\mathscr{I}$ which determines a complex structure on the tangent space $T_{z}(\mathscr{D})$ for each $z \in \mathscr{D}$. Let $k$ be the Lie algebra of $K$, and let $\mathfrak{g}=\mathfrak{k}+\mathfrak{p}$ be the corresponding Cartan decomposition of $\mathfrak{g}$. Let $z_{0} \in \mathscr{D}$ be the fixed point of $K$, and let $\mathscr{I}_{0}$ be the complex structure on $T_{z_{0}}(\mathscr{D})=\mathfrak{p}$. We set

$$
\mathfrak{p}_{ \pm}=\left\{X \in \mathfrak{p}_{\mathrm{c}} \mid \mathscr{I}_{0}(X)= \pm i X\right\},
$$

and denote by $P_{+}, P_{-}$the $\mathbb{C}$-subgroups of $G_{\mathrm{C}}$ corresponding to $\mathfrak{p}_{+}, \mathfrak{p}_{-}$, respectively. Then we have

$$
P_{+} \cap K_{\mathrm{C}} P_{-}=\{1\}, \quad G \subset P_{+} K_{\mathrm{C}} P_{-}, \quad G \cap K_{\mathrm{C}} P_{-}=K
$$

(see for example [22, Lemma II.4.2], [20]). If $g \in P_{+} K_{\mathrm{C}} P_{-} \subset G_{\mathrm{C}}$, we denote by $(g)_{+} \in P_{+},(g)_{0} \in K_{\mathrm{C}}$ and $(g)_{-} \in P_{-}$the components of $g$ such that

$$
g=(g)_{+}(g)_{0}(g)_{-} .
$$

We denote by $\left(G_{\mathrm{C}} \times \mathfrak{p}_{+}\right)_{*}$ the subset of $G_{\mathrm{C}} \times \mathfrak{p}_{+}$consisting of elements $(g, z)$ such that $g \exp z \in P_{+} K_{\mathrm{C}} P_{-}$. Then the canonical automorphy factor is the map $J$ : $\left(G_{\mathrm{C}} \times \mathfrak{p}_{+}\right)_{*} \rightarrow K_{\mathrm{C}}$ defined by

$$
J(g, z)=(g \exp z)_{0}
$$

for $(g, z) \in\left(G_{C} \times \mathfrak{p}_{+}\right)_{*}$. If $(g, z) \in\left(G_{C} \times \mathfrak{p}_{+}\right)_{*}$, we also define the element $g(z) \in \mathfrak{p}_{+}$ by

$$
\exp g(z)=(g \exp z)_{+} .
$$

Furthermore, for $z, z^{\prime} \in \mathfrak{p}_{+}$with $\left(\exp \bar{z}^{\prime}\right)^{-1} \exp z \in P_{+} K_{\mathbf{C}} P_{-}$, we set

$$
\kappa\left(z, z^{\prime}\right)=J\left(\left(\exp \bar{z}^{\prime}\right)^{-1}, z\right)^{-1}=\left(\left(\left(\exp \bar{z}^{\prime}\right)^{-1} \exp z\right)_{0}\right)^{-1} \in K_{\mathrm{C}} .
$$

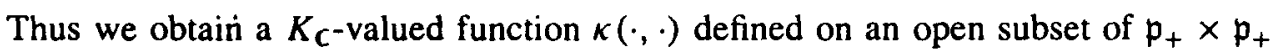
called the canonical kernel function for $G$, and it satisfies the relations

$$
\begin{gathered}
\kappa\left(z^{\prime}, z\right)={\overline{\kappa\left(z, z^{\prime}\right)}}^{-1}, \quad \kappa\left(z_{0}, z\right)=\kappa\left(z, z_{0}\right)=1, \\
\kappa\left(g(z), \bar{g}\left(z^{\prime}\right)\right)=J(g, z) \kappa\left(z, z^{\prime}\right) \overline{J(\bar{g}, w)}^{-1}
\end{gathered}
$$


for $z, z^{\prime} \in \mathfrak{p}_{+}$and $g \in G_{\mathrm{C}}$ for which $\kappa\left(z, z^{\prime}\right)$ and $\kappa\left(g(z), \bar{g}\left(z^{\prime}\right)\right)$ are defined (see for example [22, Section II.5]).

Let $(V, \beta)$ be the real symplectic space of dimension $2 n$ defined over $\mathbb{Q}$ as is described in Section 2. We extend $\beta$ to a bilinear form on $V_{C}$ and denote by $\beta_{I_{0}}$ : $V_{\mathrm{C}} \times V_{\mathrm{C}} \rightarrow \mathbb{C}$ the bilinear map defined by $\beta_{I_{0}}\left(v, v^{\prime}\right)=\beta\left(v, I_{0} v^{\prime}\right)$ for all $v, v^{\prime} \in V_{\mathrm{C}}$. Then we have $\beta_{I_{0}}=i \beta$ on $V_{+} \times V_{+}$and $\beta_{I_{0}}=-i \beta$ on $V_{-} \times V_{-}$. Thus each of $\left.\beta_{I_{0}}\right|_{V_{+}} \times V_{+}$ and $\left.\beta_{I_{0}}\right|_{V_{-} \times V_{-}}$is both symmetric and alternating; hence we have

$$
\left.\beta_{I_{0}}\right|_{V_{+} \times V_{+}}=0,\left.\quad \beta_{I_{0}}\right|_{V_{-} \times V_{-}}=0 .
$$

On the other hand, since $I_{0} \in \mathscr{H}_{n}$, from the definition of $\mathscr{H}$ it follows that both $\left.\beta_{I_{0}}\right|_{V_{+} \times V_{-}}$and $\left.\beta_{I_{0}}\right|_{V_{-} \times V_{+}}$are positive definite. Therefore we can identify $V_{-}$with the dual $V_{+}^{*}$ of $V_{+}$. Now we define a Hermitian form $\tilde{\beta}: V_{\mathbf{C}} \times V_{\mathrm{C}} \rightarrow \mathbb{C}$ on $V_{\mathrm{C}}$ by

$$
\tilde{\beta}\left(v, v^{\prime}\right)=i \beta\left(\bar{v}, v^{\prime}\right)
$$

for all $v, v^{\prime} \in V_{\mathrm{C}}$. Then $\tilde{\beta}$ is positive definite on $V_{+} \times V_{+}$, negative definite on $V_{-} \times V_{-}$, and is zero on $V_{+} \times V_{-}$and $V_{-} \times V_{+}$.

Let $\left\{u_{1}, \ldots, u_{n}\right\}$ be an orthonormal basis of $V_{+}$with respect to the restriction of the positive definite form $\tilde{\beta}$ to $V_{+} \times V_{+}$, and define the elements $u_{n+1}, \ldots, u_{2 n}$ by $u_{j+n}=\bar{u}_{j}$ for $1 \leq j \leq n$. Then we have

$$
\begin{aligned}
& i \beta\left(u_{n+j}, u_{j}\right)=i \beta\left(\bar{u}_{j}, u_{j}\right)=\tilde{\beta}\left(u_{j}, u_{j}\right)=1, \\
& i \beta\left(u_{k}, u_{n+k}\right)=i \beta\left(u_{k}, \bar{u}_{k}\right)=-i \beta\left(\bar{u}_{k}, u_{k}\right)=-\tilde{\beta}\left(u_{k}, u_{k}\right)=1
\end{aligned}
$$

for $1 \leq j, k \leq n$. On the other hand, using Lemma 1.1(iii), we obtain

$$
\begin{aligned}
i \beta\left(u_{j}, u_{k}\right) & =\tilde{\beta}\left(\bar{u}_{j}, u_{k}\right)=0, \\
i \beta\left(u_{n+j}, u_{n+k}\right) & =i \beta\left(\bar{u}_{j}, \bar{u}_{k}\right)=\tilde{\beta}\left(u_{j}, \bar{u}_{k}\right)=0
\end{aligned}
$$

for $1 \leq j, k \leq n$. Thus $\left\{u_{1}, \ldots, u_{2 n}\right\}$ is a symplectic basis for $\left(V_{\mathrm{C}}, i \beta\right)$, and we have $\operatorname{Sp}\left(V_{\mathbf{C}}, i \beta\right)=\operatorname{Sp}\left(V_{\mathbf{C}}, \beta\right)$.

Now we discuss the canonical automorphy factor for $G=\operatorname{Sp}(V, \beta)$. We shall regard the elements of $G$ and the elements of its Lie algebra as matrices using the basis $\left\{u_{1}, \ldots, u_{2 n}\right\}$ of $V_{\mathrm{C}}$ described above. Thus, for example, $\mathfrak{p}_{+}$and $P_{+}$can be written in the form

$$
\mathfrak{p}_{+}=\left\{\left(\begin{array}{ll}
0 & Z \\
0 & 0
\end{array}\right) \mid Z \in S_{n}(\mathbb{C})\right\}, \quad P_{+}=\exp \mathfrak{p}_{+}=\left\{\left(\begin{array}{ll}
1 & Z \\
0 & 1
\end{array}\right) \mid Z \in S_{n}(\mathbb{C})\right\},
$$

where $S_{n}(\mathbb{C})$ denotes the set of complex symmetric $n \times n$ matrices. Similarly, we have

$$
\mathfrak{p}_{-}=\left\{\left(\begin{array}{cc}
\frac{0}{Z} & 0 \\
0
\end{array}\right) \mid Z \in S_{n}(\mathbb{C})\right\}, \quad P_{-}=\exp \mathfrak{p}_{-}=\left\{\left(\begin{array}{cc}
\frac{1}{Z} & 0 \\
Z & 1
\end{array}\right) \mid Z \in S_{n}(\mathbb{C})\right\}
$$


We shall identify $\mathfrak{p}_{+}$with $S_{m}(\mathbb{C})$ using the correspondence $\left(\begin{array}{ll}0 & Z \\ 0 & 0\end{array}\right) \mapsto Z$. Thus we can write $\exp Z=\left(\begin{array}{ll}1 & Z \\ 0 & 1\end{array}\right) \in P_{+}$for $Z \in \mathfrak{p}_{+}=S_{m}(\mathbb{C})$, and $G_{\mathbb{C}}$ acts on $\mathfrak{p}_{+}$by

$$
g Z=\exp ^{-1}\left((g \exp Z)_{+}\right) \in \mathfrak{p}_{+}
$$

for all $g \in G_{\mathrm{C}}$, where $\exp ^{-1}(W)$ for $W \in P_{+}$denotes the $(1,2)$-block of the $2 \times 2$ block matrix $W$. Let $g^{\prime}$ be an element of $G_{\mathrm{C}}=\operatorname{Sp}\left(V_{\mathrm{C}}, \beta\right)$ whose matrix representation is of the form $g^{\prime}=\left(\begin{array}{cc}A^{\prime} & B^{\prime} \\ C & D^{\prime}\end{array}\right)$. Then $g^{\prime} \in P_{+} K_{\mathrm{C}} P_{-}$if and only if $D^{\prime}$ is nonsingular, and in this case its decomposition is given by

$$
g^{\prime}=\left(\begin{array}{cc}
1 & B^{\prime} D^{\prime-1} \\
0 & 1
\end{array}\right)\left(\begin{array}{cc}
{ }^{t} D^{\prime-1} & 0 \\
0 & D^{\prime}
\end{array}\right)\left(\begin{array}{cc}
1 & 0 \\
D^{\prime-1} C^{\prime} & 1
\end{array}\right)
$$

LEMMA 3.1. Let $g=\left(\begin{array}{cc}A & B \\ C & D\end{array}\right) \in G_{\mathrm{C}}, Z \in \mathfrak{p}_{+}=S_{n}(\mathbb{C})$, and let $J^{S}:\left(G_{\mathrm{C}} \times \mathfrak{p}_{+}\right)_{*} \rightarrow K_{\mathbf{C}}$ be the canonical automorphy factor for the symplectic group $G=\operatorname{Sp}(V, \beta)$. If $C Z+D$ is nonsingular, then we have

$$
\begin{gathered}
g Z=(A Z+B)(C Z+D)^{-1} \\
J^{s}(g, Z)=\left(\begin{array}{cc}
{ }^{t}(C Z+D)^{-1} & 0 \\
0 & (C Z+D)
\end{array}\right) .
\end{gathered}
$$

ProOF. We have

$$
g \exp Z=\left(\begin{array}{ll}
A & B \\
C & D
\end{array}\right)\left(\begin{array}{ll}
1 & Z \\
0 & 1
\end{array}\right)=\left(\begin{array}{ll}
A & A Z+B \\
C & C Z+D
\end{array}\right) .
$$

Since $C Z+D$ is nonsingular, $g \exp p$ belongs to $P_{+} K_{\mathrm{C}} P_{-}$and its decomposition is given by $g \exp Z=(g \exp Z)_{+}(g \exp Z)_{0}(g \exp Z)_{-}$,

$$
\begin{aligned}
& (g \exp Z)_{+}=\left(\begin{array}{cc}
1 & (A Z+B)(C Z+D)^{-1} \\
0 & 1
\end{array}\right), \\
& (g \exp Z)_{0}=\left(\begin{array}{cc}
{ }^{\prime}(C Z+D)^{-1} & 0 \\
0 & (C Z+D)
\end{array}\right), \\
& (g \exp Z)_{-}=\left(\begin{array}{cc}
1 & 0 \\
(C Z+D)^{-1} C & 1
\end{array}\right) .
\end{aligned}
$$

Hence it follows that

$$
J^{s}(g, Z)=(g \exp p)_{0}=\left(\begin{array}{cc}
t(C Z+D)^{-1} & 0 \\
0 & (C Z+D)
\end{array}\right)
$$

and $g Z$ is the $(1,2)$-block $(A Z+B)(C Z+D)^{-1}$ of the matrix $(g \exp p)_{+}$. 
Since the Siegel upper half space $\mathscr{H}_{n}$ can be regarded as the set of complex symmetric $n \times n$ matrices with positive definite imaginary part, there is a natural embedding of $\mathscr{H}_{n}$ into $\mathfrak{p}_{+}=S_{m}(\mathbb{C})$. If $g=\left(\begin{array}{cc}A & B \\ C & D\end{array}\right) \in G$ and $Z \in \mathscr{H}_{n}$, then $C Z+D$ is nonsingular; hence, using Lemma 3.1 we obtain the usual action $Z \mapsto(A Z+$ $B)(C Z+D)^{-1}$ of $G$ on $\mathscr{H}_{n}$. On the other hand, given $g \in G$ and $Z \in \mathscr{H}_{n}$, the associated complex $n \times n$ matrix $J^{S}(g, Z)$ can be regarded as a linear map of $V_{C}$ into itself.

COROLlaRY 3.2. Let $Z \in \mathscr{H}_{n}$ and $g=\left(\begin{array}{cc}{ }_{C}^{A} & B \\ D\end{array}\right) \in \mathrm{Sp}(V, \beta)$. Then the restriction $J_{+}^{S}(g, Z)$ of the linear map $J^{S}(g, Z): V_{\mathrm{C}} \rightarrow \mathbb{C}$ to the subspace $V_{+}$of $V_{\mathrm{C}}$ is given by

$$
J_{+}^{S}(g, Z)={ }^{t}(C Z+D)^{-1} .
$$

PROOF. This follows immediately from (3.4).

\section{Automorphy factors for generalized Jacobi groups}

In this section we describe canonical automorphy factors and canonical kernel functions of generalized Jacobi groups associated to symplectic representations of a semisimple Lie group that are equivariant with holomorphic maps of the associated symmetric domains. We shall follow closely the descriptions of Satake given in [22].

Let $\rho: G \rightarrow \operatorname{Sp}(V, \beta)$ and $\tau: \mathscr{D} \rightarrow \mathscr{H}_{n}$ be as in Section 2, and let $\widetilde{G}$ be the group of all elements of $G \times V \times \mathbb{R}$ whose multiplication operation is defined by

$$
(g, v, t)\left(g^{\prime}, v^{\prime}, t^{\prime}\right)=\left(g g^{\prime}, \rho(g) v^{\prime}+v, t+t^{\prime}+\beta\left(v, \rho(g) v^{\prime}\right) / 2\right)
$$

for all $(g, v, t),\left(g^{\prime}, v^{\prime}, t^{\prime}\right) \in G \times V \times \mathbb{R}$. Thus the subgroup $\{0\} \times V \times \mathbb{R}$ of $\widetilde{G}$ is the Heisenberg group associated to the symplectic space $(V, \beta)$. The group $\widetilde{G}$ is the group of Harish-Chandra type in the sense of Satake [22] and can be considered as a generalized Jacobi group since it reduces to a usual Jacobi group when $\rho$ is the identity map on $\operatorname{Sp}(V, \beta)$ (see for example [3, 24]). We set

$$
\tilde{\mathfrak{p}}_{+}=\mathfrak{p}_{+} \oplus V_{+}, \quad \tilde{\mathfrak{p}}_{-}=\mathfrak{p}_{+} \oplus V_{-},
$$

and let $\widetilde{P}_{+}, \widetilde{P}_{-}$be the corresponding subgroup of $\widetilde{G}_{\mathrm{C}}=G_{\mathrm{C}} \times V_{\mathrm{C}} \times \mathbb{C}$, respectively. If $\widetilde{K}_{\mathrm{C}}=K_{\mathrm{C}} \times\{0\} \times \mathbb{C}$, we have $\widetilde{G} \subset \widetilde{P}_{+} \widetilde{K}_{\mathrm{C}} \widetilde{P}_{-}$. The canonical automorphy factor $\widetilde{J}$ for the group $\widetilde{G}$ and the action of $\widetilde{G}$ on $\tilde{\mathfrak{p}}_{+}=\mathfrak{p}_{+} \oplus V_{+}$is defined by

$$
\begin{aligned}
\widetilde{J}((g, v, t),(z, w)) & =((g, v, t) \exp (z, w))_{0}, \\
\exp ((g, v, t)(z, w)) & =((g, v, t) \exp (z, w))_{+},
\end{aligned}
$$


assuming that $(g, v, t) \exp (z, w) \in \widetilde{P}_{+} \widetilde{K}_{\mathbf{C}} \widetilde{P}_{-}$. Here $\exp (z, w)$ is an element of $\widetilde{G}$ and is given by

$$
\exp (z, w)=(\exp z, w, 0)
$$

for all $z \in \mathfrak{p}_{+}$and $w \in V_{+}$. Since $\mathscr{D}$ is embedded into $\mathfrak{p}_{+}$, the quotient space $\widetilde{\mathscr{D}}=\widetilde{G} / \widetilde{K}=\mathscr{D} \times V_{+}$can be embedded into $\tilde{\mathfrak{p}}_{+}=\mathfrak{p}_{+} \oplus V_{+}$. Thus (4.3) defines the action of $\widetilde{G}$ on $\widetilde{\mathscr{D}}$. We also define the canonical kernel function $\tilde{\kappa}(\cdot, \cdot): \tilde{\mathfrak{p}}_{+} \times \tilde{\mathfrak{p}}_{+} \rightarrow \widetilde{K}_{\mathbf{C}}$ by

$$
\begin{aligned}
\tilde{\kappa}\left((z, w),\left(z^{\prime}, w^{\prime}\right)\right) & =\widetilde{J}\left(\left(\exp \overline{\left(z^{\prime}, w^{\prime}\right)}\right)^{-1},(z, w)\right)^{-1} \\
& =\left(\left(\left(\exp \overline{\left(z^{\prime}, w^{\prime}\right)}\right)^{-1} \exp (z, w)\right)_{0}\right)^{-1}
\end{aligned}
$$

for $(z, w),\left(z^{\prime}, w^{\prime}\right) \in \tilde{\mathfrak{p}}_{+}$such that

$$
\left(\exp \overline{\left(z^{\prime}, w^{\prime}\right)}\right)^{-1} \exp (z, w) \in \widetilde{P}_{+} \widetilde{K}_{\mathrm{C}} \widetilde{P}_{-} .
$$

The condition (4.6) is satisfied for $(z, w),\left(z^{\prime}, w^{\prime}\right) \in \widetilde{\mathscr{D}}$; hence we obtain a canonical kernel function on $\widetilde{\mathscr{D}} \times \widetilde{\mathscr{D}}$.

LEMMA 4.1. The canonical kernel function $\tilde{\kappa}\left((z, w),\left(z^{\prime}, w^{\prime}\right)\right)$ is holomorphic in $(z, w)$ and satisfies the relations

$$
\begin{aligned}
\tilde{\boldsymbol{\kappa}}\left(\left(z^{\prime}, w^{\prime}\right),(z, w)\right) & =\overline{\tilde{\boldsymbol{\kappa}}\left((z, w),\left(z^{\prime}, w^{\prime}\right)\right)^{-1}}, \\
\tilde{\boldsymbol{\kappa}}\left(\tilde{\boldsymbol{g}}(z, w), \tilde{\boldsymbol{g}}\left(z^{\prime}, w^{\prime}\right)\right) & =\tilde{J}(\tilde{\boldsymbol{g}},(z, w)) \tilde{\kappa}\left((z, w),\left(z^{\prime}, w^{\prime}\right)\right) \overline{\widetilde{J}\left(\tilde{\boldsymbol{g}},\left(z^{\prime}, w^{\prime}\right)\right)^{-1}}
\end{aligned}
$$

for $(z, w),\left(z^{\prime}, w^{\prime}\right) \in \widetilde{\mathscr{D}}$ and $\tilde{g} \in \widetilde{G}$.

PROOF. The first relation is immediate from the definition of $\tilde{\kappa}$. As for the second, let $(z, w),\left(z^{\prime}, w^{\prime}\right) \in \widetilde{\mathscr{D}}$ and $\tilde{g} \in \Gamma$. Then, using (4.2) and (4.3), we see that

$$
\begin{aligned}
\tilde{g} \exp (z, w) & =\exp (\tilde{g}(z, w)) \tilde{J}(\tilde{g},(z, w)) p_{1}, \\
\tilde{g} \exp \left(z^{\prime}, w^{\prime}\right) & =\exp \left(\tilde{g}\left(z^{\prime}, w^{\prime}\right)\right) \tilde{J}\left(\tilde{g},\left(z^{\prime}, w^{\prime}\right)\right) p_{2}
\end{aligned}
$$

for some $p_{1}, p_{2} \in \tilde{P}_{-}$. Hence we obtain

$$
\begin{aligned}
& \exp \overline{\left(z^{\prime}, w^{\prime}\right)^{-1}} \exp (z, w) \\
& \quad=\bar{p}_{2}^{-1} \widetilde{J\left(\tilde{g},\left(z^{\prime}, w^{\prime}\right)\right)^{-1}} \exp \overline{\left(\tilde{g}\left(z^{\prime}, w^{\prime}\right)\right)^{-1}} \exp (\tilde{g}(z, w)) \tilde{J}(\tilde{g},(z, w)) p_{1} .
\end{aligned}
$$

Thus it follows that $\exp \overline{\left(z^{\prime}, w^{\prime}\right)^{-1}} \exp (z, w) \in \widetilde{P}_{+} \widetilde{K}_{\mathrm{C}} \widetilde{P}_{-}$, and by comparing the $\widetilde{K}_{\mathrm{C}^{-}}$ components we obtain

$$
\tilde{\kappa}\left((z, w),\left(z^{\prime}, w^{\prime}\right)\right)^{-1}={\bar{J}\left(\tilde{g},\left(z^{\prime}, w^{\prime}\right)\right)^{-1}}^{-} \tilde{\kappa}\left(\tilde{g}(z, w), \tilde{g}\left(z^{\prime}, w^{\prime}\right)\right) \tilde{J}(\tilde{g},(z, w)) .
$$

Hence the proof of the lemma is complete. 
We shall now describe below the action of $\widetilde{G}$ on $\widetilde{\mathscr{D}}$ and the canonical automorphy actor for $\widetilde{G}$ defined by (4.3) more explicitly. Given $(g, v, t) \in \widetilde{G}$ and $(z, w) \in \widetilde{\mathscr{D}} \subset$ $3_{+} \oplus V_{+}$, we set

$$
\begin{aligned}
(g, v, t)(z, w) & =\left(z^{\prime}, w^{\prime}\right) \in \widetilde{\mathscr{D}} \\
\widetilde{J}((g, v, t),(z, w)) & =\left(J_{1}, 0, J_{2}\right) \in \widetilde{K}_{\mathrm{C}}=K_{\mathrm{C}} \times\{0\} \times \mathbb{C} .
\end{aligned}
$$

Hence we obtain a decomposition of the form

$$
(g, v, t) \exp (z, w)=\left(\exp z^{\prime}, w^{\prime}, 0\right)\left(J_{1}, 0, J_{2}\right)\left(p_{-}, w_{-}, 0\right)
$$

or some $\left(p_{-}, w_{-}, 0\right) \in P_{-}$. Using the multiplication rule on $\widetilde{G}$, the right-hand side ff (4.8) reduces to

$$
\left(\left(\exp z^{\prime}\right) J_{1}, w^{\prime}, J_{2}\right)\left(p_{-}, w_{-}, 0\right)=\left(g^{\prime \prime}, v^{\prime \prime}, t^{\prime \prime}\right),
$$

where

$$
\begin{aligned}
g^{\prime \prime} & =\left(\exp z^{\prime}\right) J_{1} p_{-}, \quad v^{\prime \prime}=w^{\prime}+\rho\left(\left(\exp z^{\prime}\right) J_{1}\right) w_{-}, \\
t^{\prime \prime} & =J_{2}+\beta\left(w^{\prime}, \rho\left(\left(\exp z^{\prime}\right) J_{1}\right) w_{-}\right) / 2 .
\end{aligned}
$$

In the other hand, we have

$$
\begin{aligned}
(g, v, t) \exp (z, w) & =(g, v, t)(\exp z, w, 0) \\
& =(g \exp z, v+\rho(g) w, t+\beta(v, \rho(g) w) / 2) .
\end{aligned}
$$

Hence we obtain

$$
\begin{aligned}
g \exp z & =\left(\exp z^{\prime}\right) J_{1} p_{-}, \\
v+\rho(g) w & =w^{\prime}+\left(\exp z^{\prime}\right) J_{1} w_{-}, \\
t+\beta(v, \rho(g) w) / 2 & =J_{2}+\beta\left(w^{\prime}, \rho\left(\left(\exp z^{\prime}\right) J_{1}\right) w_{-}\right) / 2
\end{aligned}
$$

ior $(g, v, t) \in \widetilde{G}=G \times V \times \mathbb{R}$ and $(z, w) \in \widetilde{\mathscr{D}}=\mathscr{D} \times V_{+}$.

Proposition 4.2. For $(z, w) \in \widetilde{\mathscr{D}}$ we set $\rho\left(J_{1}(z, w)\right)=\left(\begin{array}{cc}J_{+}(z, w) & 0 \\ 0 & J_{-}(z, w)\end{array}\right)$. Then the xction of $\widetilde{G}$ on $\widetilde{\mathscr{D}}$ is given by

$$
(g, v, t)(z, w)=\left(g(z), v_{g z}+J_{+}(z, w) w\right)
$$

for all $(g, v, t) \in \widetilde{G}$ and $(z, w) \in \widetilde{\mathscr{D}}$.

Proof. Consider the elements $(g, v, t) \in \widetilde{G}$ and $(z, w) \in \widetilde{\mathscr{D}}$, and assume that $(g, v, t)(z, w)=\left(z^{\prime}, w^{\prime}\right) \in \widetilde{\mathscr{D}}$ as in (4.7). Since $\exp g(z)=(g \exp z)_{+}$by (3.2), from (4.9) we obtain $\exp z^{\prime}=\exp g(z)$ and $J_{1}=(g \exp z)_{-}$. Hence it follows that 
$z^{\prime}=g(z)$, and $J_{1}((g, v, t),(z, w))=J(g, z)$, where $J$ is the canonical automorphy factor for the group $G$ given in (3.1). Now we consider the matrix representations

$$
\rho(\exp z)=\left(\begin{array}{cc}
1 & \tau(z) \\
0 & 1
\end{array}\right), \quad \rho(g)=\left(\begin{array}{cc}
A & B \\
C & D
\end{array}\right), \quad \rho\left(J_{1}\right)=\left(\begin{array}{cc}
J_{+} & 0 \\
0 & J_{-}
\end{array}\right)
$$

for $z \in \mathfrak{p}_{-}$relative to the decomposition $V=V_{+} \oplus V_{-}$. Applying $\rho$ to both sides of the relation (4.9), we obtain

$$
\left(\begin{array}{ll}
A & B \\
C & D
\end{array}\right)\left(\begin{array}{cc}
1 & \tau(z) \\
0 & 1
\end{array}\right)=\left(\begin{array}{cc}
1 & \tau\left(z^{\prime}\right) \\
0 & 1
\end{array}\right)\left(\begin{array}{cc}
J_{+} & 0 \\
0 & J_{-}
\end{array}\right)\left(\begin{array}{cc}
1 & 0 \\
M & 1
\end{array}\right)
$$

for some matrix $M$. Thus we have

$$
\left(\begin{array}{ll}
A & A \tau(z)+B \\
C & C \tau(z)+D
\end{array}\right)=\left(\begin{array}{cc}
J_{+}+\tau\left(z^{\prime}\right) J_{-} M & \tau\left(z^{\prime}\right) J_{-} \\
J_{-} M & J_{-}
\end{array}\right) .
$$

Hence we see that

$$
\begin{gathered}
J_{+}=A-\tau\left(z^{\prime}\right) C, \quad J_{-}=C \tau(z)+D, \\
\tau\left(z^{\prime}\right)=(A \tau(z)+B)(C \tau(z)+D)^{-1} .
\end{gathered}
$$

On the other hand, the matrix form of the relation (4.10) is given by

$$
\left(\begin{array}{l}
v_{+} \\
v_{-}
\end{array}\right)+\left(\begin{array}{ll}
A & B \\
C & D
\end{array}\right)\left(\begin{array}{c}
w \\
0
\end{array}\right)=\left(\begin{array}{c}
w^{\prime} \\
0
\end{array}\right)+\left(\begin{array}{cc}
1 & \tau\left(z^{\prime}\right) \\
0 & 1
\end{array}\right)\left(\begin{array}{cc}
J_{+} & 0 \\
0 & J_{-}
\end{array}\right)\left(\begin{array}{c}
0 \\
w_{-}
\end{array}\right)
$$

which implies that $v_{+}+A w=w^{\prime}+\tau\left(z^{\prime}\right) J_{-} w_{-}$and $v_{-}+C w=J_{-} w_{-}$. Therefore we obtain

$$
\begin{aligned}
w^{\prime} & =\left(v_{+}+A w\right)-\tau\left(z^{\prime}\right)\left(v_{-}+C w\right) \\
& =\left(v_{+}-\tau\left(z^{\prime}\right) v_{-}\right)+\left(A-\tau\left(z^{\prime}\right) C\right) w=v_{z^{\prime}}+J_{+} w
\end{aligned}
$$

hence we have $\left(z^{\prime}, w^{\prime}\right)=\left(g(z), v_{g z}+J_{+} w\right)$.

COROLLARY 4.3. Let $J_{+}^{s}$ be the restriction of the canonical automorphy factor for $\operatorname{Sp}(V, \beta)$ to $V_{+}$given in Corollary 3.2. Then, for $(g, v, t) \in \widetilde{G}$ and $(z, w) \in \widetilde{\mathscr{D}}$, we have

$$
(g, v, t)(z, w)=\left(g(z), v_{g z}+J_{+}^{S}(\rho(g), \tau(z)) w\right) .
$$

In particular, if $\rho(g)=\left(\begin{array}{cc}A_{\rho} & B_{\rho} \\ C_{p} & D_{\rho}\end{array}\right) \in \mathrm{Sp}(V, \beta)$, then we have

$$
(g, v, t)(z, w)=\left(g z, v_{g z}+{ }^{\prime}\left(C_{\rho} \tau(z)+D_{\rho}\right)^{-1} w\right) .
$$


PROOF. From (3.1), for $g \in G$ and $z \in \mathscr{D}$, we have

$$
\rho\left(J_{1}\right)=\rho\left(J_{1}(g, z)\right)=\rho\left((g \exp z)_{0}\right)=(\rho(g) \exp \tau(z))_{0} .
$$

Thus we see that $\rho\left(J_{1}\right)(g, z)=J^{s}(\rho(g), \tau(z))$, where $J^{s}$ is the canonical automorphy factor for the symplectic group $\operatorname{Sp}(V, \beta)$ given in (3.4). Therefore, if $J_{+}$is as in Proposition 4.2, we have

$$
J_{+}(g, z)=J^{s}(\rho(g), \tau(z)) .
$$

Using this and Proposition 4.2, we obtain (4.13). Then (4.14) is obtained by using (3.5).

From the multiplication operation on $\widetilde{G}$ given in (4.1) we see that the induced operation on $G \times V$ by the natural projection $\widetilde{G} \rightarrow G \times V$ is exactly the one on $G \ltimes_{\rho} V$ considered in Section 2. On the other hand, we considered an operation of $G \ltimes_{\rho} V$ on $\mathscr{D} \times V_{+}$in (2.4) to construct a Kuga fiber variety. This operation is in fact compatible with the operation of $\widetilde{G}$ given in (4.11) as can be seen in the next lemma.

LEMMA 4.4. The operation of $G \ltimes_{\rho} V$ on $\mathscr{D} \times V_{+}$given in (2.4) can be written in the form $(g, v)(z, w)=\left(g z, v_{g z}+J_{+} w\right)$ for all $(g, v) \in G \ltimes_{\rho} V$ and $(z, w) \in \widetilde{\mathscr{D}}=\mathscr{D} \times V_{+}$.

PROOF. Let $(g, v) \in G \ltimes_{\rho} V$ and $(z, w) \in \mathscr{D} \times V_{+}$. From (2.4), we have

$$
(g, v)(z, w)=\left(g z, \xi(g z, \rho(g) u)+v_{g z}\right),
$$

where $u$ is an element of $\left(V, I_{\tau(z)}\right)$ such that $w=\xi(z, u)$. Using $(2.1)$ and $\rho(g)=$ $\left(\begin{array}{ll}A & B \\ C & D\end{array}\right)$, we have

$$
\begin{aligned}
\xi(g z, \rho(g) u) & =(\rho(g) u)_{g z}=\left(\left(\begin{array}{ll}
A & B \\
C & D
\end{array}\right)\left(\begin{array}{l}
u_{+} \\
u_{-}
\end{array}\right)\right)_{g z} \\
& =\left(A u_{+}+B u_{-}\right)-\tau(g z)\left(C u_{+}+D u_{-}\right) \\
& =(A-\tau(g z) C) u_{+}+(B-\tau(g z) D) u_{-} .
\end{aligned}
$$

However, we have

$$
\begin{aligned}
\tau(z) & =\rho(g)^{-1} \tau(g z)=\left(\begin{array}{cc}
{ }^{t} D & -{ }^{t} B \\
-{ }^{t} C & { }^{t} A
\end{array}\right) \tau(g z) \\
& =\left({ }^{t} D \tau(g z)-{ }^{t} B\right)\left(-{ }^{t} C \tau(g z)+{ }^{t} A\right)^{-1} .
\end{aligned}
$$

Using this and the fact that $\tau(g z)$ is symmetric, we obtain

$$
B-\tau(g z) D=-(A-\tau(g z) C) \tau(z) .
$$


Hence we see that

$$
\xi(g z, \rho(g) u)=(A-\tau(g z) C)\left(u_{+}-\tau(z) u_{-}\right)=(A-\tau(g z) C) w .
$$

Since $A-\tau(g z) C=J_{+}$by (4.12), we obtain the desired formula.

COROLlaRY 4.5. Let $C_{\rho}, D_{\rho}$ and $J_{+}^{S}$ be as in Corollary 4.3. Then the operation of $G \ltimes_{\rho} V$ on $\mathscr{D} \times V_{+}$given in (2.4) can be written in the form

$$
\begin{aligned}
(g, v)(z, w) & =\left(g z, v_{g z}+J_{+}^{S}(\rho(g), \tau(z)) w\right) \\
& =\left(g z, v_{g z}+{ }^{t}\left(C_{\rho} \tau(z)+D_{\rho}\right)^{-1} w\right) .
\end{aligned}
$$

PrOOF. This follows from (3.5), (4.15) and Lemma 4.4.

LEMMA 4.6. If $(g, v, t)(z, w)=\left(z^{\prime}, w^{\prime}\right)$, then we have

$$
\beta(v, \rho(g) w)-\beta\left(v_{z^{\prime}}, C w\right)=\beta\left(v, J_{1} w\right),
$$

where the matrix $C$ is the $(2,1)$ block of $\rho(g)$ as in the proof of Lemma 4.2.

PROoF. Using $v_{z^{\prime}}=v_{+}-\tau\left(z^{\prime}\right) v_{-}$and the matrix representation of $\rho(g)$, we have

$$
\beta(v, \rho(g) w)-\beta\left(v_{z^{\prime}}, C w\right)=\beta(v, A w+C w)-\beta\left(\left(\begin{array}{cc}
1 & -\tau\left(z^{\prime}\right) \\
0 & 1
\end{array}\right) v+v_{-}, C w\right) .
$$

Since $C w \in V_{-}$and $\left.\beta\right|_{V_{-} \times V_{-}}=0$, using the fact that $\beta$ is invariant under $\rho(G)$, we obtain

$$
\begin{aligned}
\beta(v, \rho(g) w)-\beta\left(v_{z^{\prime}}, C w\right) & =\beta(v, A w)+\beta(v, C w)-\beta\left(v,\left(\begin{array}{cc}
1 & \tau\left(z^{\prime}\right) \\
0 & 1
\end{array}\right) C w\right) \\
& =\beta(v, A w)+\beta(v, C w)-\beta\left(v, C w+\tau\left(z^{\prime}\right) C w\right) \\
& =\beta\left(v, A w-\tau\left(z^{\prime}\right) C w\right) .
\end{aligned}
$$

Now the lemma follows from the fact that $J_{1} w=J_{+} w=A w-\tau\left(z^{\prime}\right) C w$.

PROPOSITION 4.7. The canonical automorphy factor $\widetilde{J}$ for $\widetilde{G}$ is given by

$$
\widetilde{J}((g, v, t),(z, w))=\left(J_{1}((g, v),(z, w)), 0, J_{2}((g, v, t),(z, w))\right)
$$

for all $(g, v, t) \in \widetilde{G}$ and $(z, w) \in \widetilde{\mathscr{D}}$, where $J_{1}$ is the canonical automorphy factor for the group $G$ and

$$
J_{2}((g, v, t),(z, w))=t+\beta\left(v, v_{g z}\right) / 2+\beta\left(v, J_{1} w\right)+\beta\left(\rho(g) w, J_{1} w\right) / 2 .
$$


Proof. If $C$ is as in Lemma 4.6, then we have $(v+\rho(g) w)_{-}=v_{-}+C w$. Using this and the fact that $\left.\beta\right|_{V_{+} \times V_{+}}=0$, we see that

$$
\begin{aligned}
J_{2}= & t+\beta(v, \rho(g) w) / 2-\beta\left(w^{\prime}, \rho\left(\left(\exp z^{\prime}\right) J_{1}\right) w_{-}\right) / 2 \\
= & t+\beta(v, \rho(g) w) / 2-\beta\left(w^{\prime}, v+\rho(g) w-w^{\prime}\right) / 2 \\
= & t+\beta(v, \rho(g) w) / 2-\beta\left(v_{z^{\prime}}+J_{+} w, v+\rho(g) w\right) / 2 \\
= & t+\beta(v, \rho(g) w) / 2-\beta\left(v_{z^{\prime}}, v\right) / 2-\beta\left(v_{z^{\prime}}, \rho(g) w\right) / 2-\beta\left(J_{+} w, v_{-}+C w\right) / 2 \\
= & t+\beta(v, \rho(g) w) / 2-\beta\left(v_{z^{\prime}}, v\right) / 2-\beta\left(v_{z^{\prime}}, \rho(g) w\right) / 2 \\
& -\beta\left(J_{+} w, v_{-}\right) / 2-\beta\left(J_{+} w, C w\right) / 2 .
\end{aligned}
$$

Using Lemma 4.6, we thus obtain

$$
\begin{aligned}
J_{2} & =t+\beta\left(v, J_{1} w\right) / 2-\beta\left(v_{z^{\prime}}, v\right) / 2-\beta\left(J_{+} w, v_{-}\right) / 2-\beta\left(J_{+} w, C w\right) / 2 \\
& =t+\beta\left(v, J_{1} w\right)-\beta\left(v_{z^{\prime}}, v\right) / 2-\beta\left(J_{+} w, C w\right) / 2 .
\end{aligned}
$$

Since $\rho(g) w=A w+C w$ with $A w \in V_{+}$, we have

$$
\beta\left(J_{+} w, C w\right)=\beta\left(J_{+} w, \rho(g) w\right)=\beta\left(J_{1} w, \rho(g) w\right)=-\beta\left(\rho(g) w, J_{1} w\right) .
$$

Hence the proposition follows using this and $\beta\left(v_{z^{\prime}}, v\right)=\beta\left(v_{g z}, v\right)=-\beta\left(v, v_{g z}\right)$.

Now we define the complex valued function $\mathscr{J}: \widetilde{G} \times \widetilde{\mathscr{D}} \rightarrow \mathbb{C}$ by

$$
\begin{aligned}
\mathscr{J}((g, v, t),(z, w)) & =\mathbf{e}\left[J_{2}((g, v, t),(z, w))\right] \\
& =\mathbf{e}\left[t+\beta\left(v, v_{g z}\right) / 2+\beta\left(v, J_{1} w\right)+\beta\left(\rho(g) w, J_{1} w\right) / 2\right]
\end{aligned}
$$

for all $(g, v, t) \in \widetilde{G}$, where $\mathbf{e}[\cdot]=e^{2 \pi i(\cdot)}$.

PROPOSITION 4.8. The function $\mathscr{J}$ is an automorphy factor, that is, it satisfies the relation

$$
\mathscr{J}\left(\tilde{g} \tilde{g}^{\prime}, \tilde{z}\right)=\mathscr{J}\left(\tilde{g}, \tilde{g}^{\prime} \tilde{z}\right) \mathscr{J}(\tilde{g}, \tilde{z})
$$

for $\tilde{g}=(g, v, t), \tilde{g}^{\prime}=\left(g^{\prime} \cdot v^{\prime}, t^{\prime}\right) \in \widetilde{G}$ and $\tilde{z}=(z, w) \in \widetilde{\mathscr{D}}$.

PROOF. Let $\tilde{g}, \tilde{g}^{\prime} \in \widetilde{G}$ and $\tilde{z} \in \widetilde{\mathscr{D}}$. Since the map $\widetilde{J}=\left(J_{1}, 0, J_{2}\right): \widetilde{G} \times \widetilde{\mathscr{D}} \rightarrow \widetilde{K}_{\mathrm{C}}$ is an automorphy factor, using the multiplication rule (4.1) in $\widetilde{G}$, we obtain

$$
\begin{aligned}
\left(J_{1}\left(\tilde{g} \tilde{g}^{\prime}, \tilde{z}\right), 0, J_{2}\left(\tilde{g} \tilde{g}^{\prime}, \tilde{z}\right)\right) & =\left(J_{1}\left(\tilde{g}, \tilde{g}^{\prime} \tilde{z}\right), 0, J_{2}\left(\tilde{g}, \tilde{g}^{\prime} \tilde{z}\right)\right)\left(J_{1}\left(\tilde{g}^{\prime}, \tilde{z}\right), 0, J_{2}\left(\tilde{g}^{\prime}, \tilde{z}\right)\right) \\
& =\left(J_{1}\left(\tilde{g}, \tilde{g}^{\prime} \tilde{z}\right) J_{1}\left(\tilde{g}^{\prime}, \tilde{z}\right), 0, J_{2}\left(\tilde{g}, \tilde{g}^{\prime} \tilde{z}\right)+J_{2}\left(\tilde{g}^{\prime}, \tilde{z}\right)\right) .
\end{aligned}
$$

Thus we have $J_{2}\left(\tilde{g} \tilde{g}^{\prime}, \tilde{z}\right)=J_{2}\left(\tilde{g}, \tilde{g}^{\prime} \tilde{z}\right)+J_{2}\left(\tilde{g}^{\prime}, \tilde{z}\right)$, and hence $\mathscr{J}=\mathbf{e}\left[J_{2}\right]$ satisfies the desired relation. 


\section{Twisted torus bundles and Jacobi forms}

In this section, we construct twisted torus bundles over locally symmetric spaces, ol circle bundles over Kuga fiber varieties, associated to generalized Jacobi groups. We then define Jacobi forms associated to an equivariant pair, which generalize the usua] Jacobi forms (see [24]), and discuss connections between such generalized Jacobi forms and twisted torus bundles. Similar Jacobi forms were also considered in [17] and [18].

Let $\widetilde{G}=G \times V \times \mathbb{R}$ be as in Section 4. Thus we have $\widetilde{G} / \widetilde{K}=\widetilde{\mathscr{D}}=\mathscr{D} \times V_{+}$, and there are embeddings $\widetilde{\mathscr{D}} \hookrightarrow \tilde{\mathfrak{p}}_{+}=\mathfrak{p}_{+} \oplus V_{+} \hookrightarrow \widetilde{G}_{\mathrm{C}} / \widetilde{K}_{\mathrm{C}} \widetilde{P}_{-}$. On the other hand, since the elements of $\exp \mathbb{C}$ and the elements of $\widetilde{P}_{+}$commute, the exponential map determines the natural embedding $\tilde{\mathfrak{p}}_{+} \oplus \mathbb{C}=\mathfrak{p}_{+} \oplus V_{+} \oplus \mathbb{C} \hookrightarrow \widetilde{G}_{\mathbf{C}} / K_{\mathbf{C}} \widetilde{P}_{-}$that induces the embedding $\tilde{\mathfrak{p}}_{+} \hookrightarrow \widetilde{G}_{\mathrm{C}} / \widetilde{K}_{\mathbf{C}} \widetilde{P}_{-}$. Thus we obtain a commutative diagram

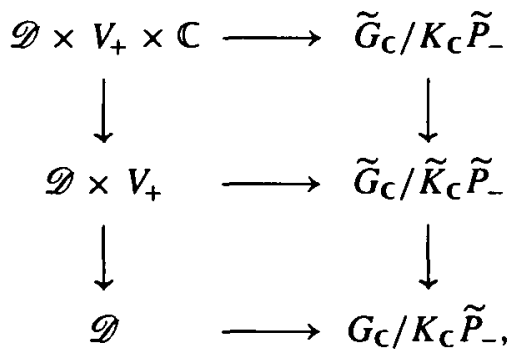

where the horizontal arrows are the natural embeddings and the vertical arrows are the natural projection maps. Now we can define an action of $\widetilde{G}$ on $\mathscr{D} \times V_{+} \times \mathbb{C}$ by requiring that $(g, v, t)(z, w, u)=\left(z^{\prime}, w^{\prime}, u^{\prime}\right)$ if and only if

$$
(g, v, t) \exp (z, w, u) \in \exp \left(z^{\prime}, w^{\prime}, u^{\prime}\right) K_{\mathrm{C}} \widetilde{P}_{-} .
$$

More specifically, we define the action by

$$
(g, v, t) \exp (z, w, u)=\exp \left(z^{\prime}, w^{\prime}, u^{\prime}\right) J_{1} p_{-}
$$

for all $(g, v, t) \in \widetilde{G}$ and $(z, w, u) \in \mathscr{D} \times V_{+} \times \mathbb{C}$, where $J_{1} \in K_{\mathrm{C}}$ is the canonical automorphy factor for the group $G$ and $p_{-}$is an element of $P_{-}$. By considering the natural projection $\mathscr{D} \times V_{+} \times \mathbb{C} \rightarrow \mathscr{D} \times V_{+}$, we obtain

$$
(g, v, t) \exp (z, w)=\exp \left(z^{\prime}, w^{\prime}\right) \exp \left(u^{\prime}-u\right) J_{1} p_{-},
$$

which implies that $((g, v, t) \exp (z, w))_{0}=\exp \left(u^{\prime}-u\right) J_{1}=\left(u^{\prime}-u, 0, J_{1}\right)$. However, using (4.2) and (4.16), we see that

$$
((g, v, t) \exp (z, w))_{0}=\left(J_{2}, 0, J_{1}\right) .
$$


Therefore we obtain $u^{\prime}=u+J_{2}$, hence it follows that

$$
\begin{gathered}
(g, v, t)(z, w, u)=\left(g z, v_{g z}+J_{+} w, u+J_{2}\right), \\
J_{2}=t+\beta\left(v, v_{g z}\right) / 2+\beta\left(v, J_{1} w\right)+\beta\left(\rho(g) w, J_{1} w\right) / 2 .
\end{gathered}
$$

Now we restrict the holomorphic action of $\widetilde{G}$ on $\mathscr{D} \times V_{+} \times \mathbb{C}$ to the real analytic action of $\widetilde{G}$ on $\mathscr{D} \times V_{+} \times \mathbb{R}$. Let the arithmetic subgroup $\Gamma \subset G$ and the lattice $L \subset V_{+}$be as in Section 2, and consider the quotient $\mathscr{T}=\Gamma \times L \times \mathbb{Z} \backslash \mathscr{D} \times V_{+} \times \mathbb{R}$ of $\mathscr{D} \times V_{+} \times \mathbb{R}$ by the action of $\Gamma \times L \times \mathbb{Z} \subset \widetilde{G}$ given in (5.1). We shall identify $\mathscr{T}$ with the quotient $\Gamma \times L \backslash \mathscr{D} \times V_{+} \times(\mathbb{R} / \mathbb{Z})$ by using the map

$$
(z, w, u) \mapsto(z, w, \mathbf{e}[u]), \quad \mathscr{D} \times V_{+} \times \mathbb{R} \rightarrow \mathscr{D} \times V_{+} \times(\mathbb{R} / \mathbb{Z}),
$$

where we identify $\mathbb{R} / \mathbb{Z}$ with the unit circle $\{z \in \mathbb{C}|| z \mid=1\}$ in $\mathbb{C}$. Then the action of $\Gamma \times L$ on $\mathscr{D} \times V_{+} \times(\mathbb{R} / \mathbb{Z})$ is given by

$$
(\gamma, l)(z, w, \lambda)=\left(\gamma z, l_{\gamma z}+J_{+} w, \mathscr{J}((\gamma, l, 0),(z, w)) \lambda\right)
$$

for all $(\gamma, l) \in \Gamma \times L$ and $(z, w, \lambda) \in \mathscr{D} \times V_{+} \times(\mathbb{R} / \mathbb{Z})$, and the natural projection map $\mathscr{D} \times V_{+} \times(\mathbb{R} / \mathbb{Z}) \rightarrow \mathscr{D} \times V_{+}$equips $\mathscr{T}$ with the structure of a fiber bundle $\pi^{\prime}: \mathscr{T} \rightarrow Y$ over the Kuga fiber variety $Y=\Gamma \times L \backslash \mathscr{D} \times V_{+}$whose fiber is isomorphic to the circle $\mathbb{R} / \mathbb{Z}$. By composing with $\pi: Y \rightarrow X$ we can also consider $\mathscr{T}$ as a twisted torus bundle over the arithmetic variety $X=\Gamma \backslash \mathscr{D}$ in the sense of [15].

DEFINITION 5.1. A holomorphic function $f: \mathscr{D} \times V_{+} \rightarrow \mathbb{C}$ is a Jacobi form of weight $\mu$ and index $v$ for $(\Gamma, \rho, \tau)$ if it satisfies the relation

$$
\begin{aligned}
f\left(\gamma z, l_{\gamma z}+J_{+} w\right)= & \operatorname{det}\left(C_{\rho} \tau(z)+D_{\rho}\right)^{\mu} \mathbf{e}^{\nu}\left[\beta\left(l, l_{\gamma z}\right) / 2\right. \\
& \left.+\beta\left(l, J_{1} w\right)+\beta\left(\rho(\gamma) w, J_{1} w\right) / 2\right] f(z, w)
\end{aligned}
$$

for all $(z, w) \in \mathscr{D} \times V_{+}$and $(\gamma, l) \in \Gamma \times L$ with

$$
\rho(\gamma)=\left(\begin{array}{ll}
A_{\rho} & B_{\rho} \\
C_{\rho} & D_{\rho}
\end{array}\right) \in \mathrm{Sp}(V, \beta),
$$

where $\mathbf{e}^{\nu}[*]=\mathbf{e}[v(*)]=e^{2 \pi i v(*)}$.

Let $\mathscr{L}$ be the line bundle on $Y=\Gamma \times L \backslash \mathscr{D} \times V_{+}$defined by

$$
\mathscr{L}=\Gamma \times L \backslash \mathscr{D} \times V_{+} \times \mathbb{C},
$$

where the quotient is taken with respect to the action of $\Gamma \times L$ on $\mathscr{D} \times V_{+} \times \mathbb{C}$ given by

$$
(\gamma, l)(z, w, \zeta)=\left(\gamma z, l_{\gamma z}+J_{+} w, \operatorname{det}\left(C_{\rho} \tau(z)+D_{\rho}\right) \zeta\right)
$$

for all $(z, w, \zeta) \in \mathscr{D} \times V_{+} \times \mathbb{C}$ and $(\gamma, l) \in \Gamma \times L$ with $\rho(g)$ as in Definition 5.1. 
THEOREM 5.2. The space of Jacobi forms of weight $\mu$ and index $\nu$ for $(\Gamma, \rho, \tau)$ is isomorphic to the space $\Gamma_{0}\left(Y, \mathscr{L}^{\otimes \mu} \otimes \mathscr{T}^{\otimes v}\right)$ of sections of the bundle $\mathscr{L}^{\otimes \mu} \otimes \mathscr{T}^{\otimes v}$ over the Kuga fiber variety $Y=\Gamma \times L \backslash \mathscr{D} \times V_{+}$.

ProOF. From (5.2) and (5.5) it follows that the line bundle $\mathscr{L}^{\otimes \mu} \otimes \mathscr{T}^{\otimes v}$ can be regarded as the quotient $\mathscr{L}^{\otimes \mu} \otimes \mathscr{T}^{\otimes \nu}=\Gamma \times L \backslash \mathscr{D} \times V_{+} \times \mathbb{C}$ with respect to the action of $\Gamma \times L$ on $\mathscr{D} \times V_{+} \times \mathbb{C}$ given by

$$
(\gamma, l)(z, w, \zeta)=\left(\gamma z, l_{\gamma z}+J_{+} w, \operatorname{det}\left(C_{\rho} \tau(z)+D_{\rho}\right)^{\mu} \mathscr{J}((\gamma, l, 0),(z, w))^{\nu} \zeta\right) .
$$

Let $s: Y \rightarrow L^{\otimes \mu} \otimes \mathscr{T}^{\otimes v}$ be an element of $\Gamma_{0}\left(Y, \mathscr{L}^{\otimes \mu} \otimes \mathscr{T}^{\otimes v}\right)$. Then for $(z, w) \in \mathscr{D} \times V_{+}$we have $s([(z, w)])=\left[\left(z, w, \zeta_{(z, w)}\right)\right]$ for some $\zeta_{(z, w)} \in \mathbb{R} / \mathbb{Z}$, where $[(\cdot)]$ denotes the appropriate coset corresponding to the element $(\cdot)$. We define the function $f_{s}: \mathscr{D} \times V_{+} \rightarrow \mathbb{C}$ by $f_{s}(z, w)=\zeta_{(z, w)}$ for all $(z, w) \in \mathscr{D} \times V_{+}$. For each $(\gamma, l) \in \Gamma \times L$, using the actions of $\Gamma \times L$ given in (5.2) and (5.5), we have

$$
\begin{aligned}
s([(z, w)]) & =s\left(\left[\left(\gamma z, l_{\gamma z}+J_{+} w\right)\right]\right) \\
& =\left[\left(\gamma z, l_{y z}+J_{+} w, \zeta_{\left(\gamma z, l_{\gamma z}+J_{+} w\right)}\right)\right] \\
& =\left[(\gamma, l)^{-1}\left(\gamma z, l_{\gamma z}+J_{+} w, \zeta_{\left(\gamma z, l_{z}+J_{+} w\right)}\right)\right] \\
& =\left[\left(z, w, \operatorname{det}\left(C_{\rho} \tau(z)+D_{\rho}\right)^{-\mu} \mathscr{J}((\gamma, l, 0),(z, w))^{-\nu} \zeta_{\left(\gamma z, l_{z z}+J_{+} w\right)}\right)\right],
\end{aligned}
$$

where $C_{\rho}$ and $D_{\rho}$ are as in (5.4) and, as in (4.17),

$$
\begin{aligned}
\mathscr{J}((\gamma, l, 0),(z, w)) & =\mathbf{e}\left[J_{2}((\gamma, l, 0),(z, w))\right] \\
& =\mathbf{e}\left[\beta\left(l, l_{\gamma z}\right) / 2+\beta\left(l, J_{1} w\right)+\beta\left(\rho(\gamma) w, J_{1} w\right) / 2\right] .
\end{aligned}
$$

Therefore we obtain

$$
f_{s}(z, w)=\operatorname{det}\left(C_{\rho} \tau(z)+D_{\rho}\right)^{-\mu} \mathscr{J}((\gamma, l, 0),(z, w))^{-v} f_{s}\left(\gamma z, l_{\gamma z}+J_{+} w\right) .
$$

Hence $f_{s}$ satisfies the transformation formula (5.3) for a Jacobi form of weight $\mu$ and index $\nu$ for $(\Gamma, \rho, \tau)$. On the other hand, suppose that $f: \mathscr{D} \times V_{+} \rightarrow \mathbb{C}$ is a holomorphic function satisfying the condition (5.3). We define the map $s_{f}: Y \rightarrow$ $L^{\otimes \mu} \otimes \mathscr{T}^{\otimes v}$ by

$$
s_{f}([(z, w)])=[(z, w, f(z, w))]
$$

for $(z, w) \in \mathscr{D} \times V_{+}$. Then this map is well-defined because, for each $(\gamma, l) \in \Gamma \times L$, we have

$$
\begin{aligned}
& s_{f}([(\gamma, l)(z, w)]) \\
& \quad=s_{f}\left(\left[\left(\gamma z, l_{\gamma z}+J_{+} w\right)\right]\right)=\left[\left(\gamma z, l_{\gamma z}+J_{+} w, f\left(\gamma z, l_{\gamma z}+J_{+} w\right)\right)\right] \\
& \quad=\left[\left(\gamma z, l_{\gamma z}+J_{+} w, \operatorname{det}\left(C_{\rho} \tau(z)+D_{\rho}\right)^{\mu} \mathscr{J}((\gamma, l, 0),(z, w))^{\nu} f(z, w)\right.\right. \\
& \quad=[(\gamma, l)((z, w, f(z, w))]=[(z, w, f(z, w))],
\end{aligned}
$$


which is equal to $s_{f}([(z, w)])$ in (5.7). Thus $s_{f}$ is a section of $\mathscr{L}^{\otimes \mu} \otimes \mathscr{T}^{\otimes \nu}$, and the proof of the theorem is complete.

EXAMPLE 5.3. Let $W$ be a real vector space of dimension $\nu>2$ defined over $\mathbb{Q}$, and let $S$ be a nondegenerate symmetric bilinear form of signature $(2, v-2)$. Then it is known (see [22]) that the associated spin group $G=\operatorname{Spin}(W, S)$ is a semisimple Lie group of Hermitian type. Let $\mathscr{C}^{+}$be the even part of the Clifford algebra $\mathscr{C}$ of $(W, S)$, and let $a \in \mathscr{C}^{+}$be an element with $a^{\iota}=-a$, where $\iota$ is the canonical involution of $\mathscr{C}$. Then the bilinear form $A$ on $\mathscr{C}^{+}$with $A(x, y)=\operatorname{tr}\left(a x^{t} y\right)$ is nondegenerate and alternating, and for each $x \in G$ the map $x \mapsto g x$ determines an element of $\operatorname{Sp}\left(\mathscr{C}^{+}, A\right)$. By identifying $\operatorname{Sp}\left(\mathscr{C}^{+}, A\right)$ with $\operatorname{Sp}\left(2^{\nu}, \mathbb{R}\right)$ we obtain a homomorphism $\rho: \operatorname{Spin}(W, S) \rightarrow \operatorname{Sp}\left(2^{\nu}, \mathbb{R}\right)$, which induces an equivariant holomorphic map $\tau: \mathscr{D} \rightarrow \mathscr{H}_{2^{v}}$ of the symmetric domain $\mathscr{D}$ associated to $G$ into the Siegel upperhalf space $\mathscr{H}_{2^{v}}$. Thus we obtain Jacobi form on the symmetric domain associated to spin groups of type $(2, n)$, and such Jacobi forms were studied recently in connection with a number of topics (see for example [4] and [7]).

\section{Fock representations}

Let $\widetilde{G}=G \times V \times \mathbb{R}$ be as in Section 4. Then the multiplication operation (4.1) restricted to the subgroup $\{1\} \times V \times \mathbb{R} \cong V \times \mathbb{R}$ of $\widetilde{G}$ is the usual multiplication operation on the Heisenberg group $V \times \mathbb{R}$. Classically a Fock representation of such a Heisenberg group is a representation in a Hilbert space of certain functions on $V_{\mathbf{C}}$ associated to a point in the corresponding Siegel upper half space (see [21]). In this section we construct similar representations of such a Heisenberg group in Hilbert spaces associated to points in the Hermitian symmetric domain $\mathscr{D}$ and prove that such representations are unitary and irreducible.

Let $\tilde{\kappa}(\cdot, \cdot)$ be the canonical kernel function for the group $\widetilde{G}=G \times V \times \mathbb{R}$ given in (4.5). Thus we have $\tilde{\kappa}\left((z, w),\left(z^{\prime}, w^{\prime}\right)\right)=\tilde{J}\left(\left(\exp \overline{\left(z^{\prime}, w^{\prime}\right)}\right)^{-1},(z, w)\right)^{-1}$ for $(z, w),\left(z^{\prime}, w^{\prime}\right) \in \widetilde{\mathscr{D}}=\mathscr{D} \times V_{+}$. Since $\widetilde{J}=\left(J_{1}, 0, J_{2}\right) \in \widetilde{K}_{\mathrm{C}}$, by restricting $\tilde{\kappa}$ to $\mathscr{D} \times \mathscr{D}$ we have

$$
\begin{aligned}
\tilde{\kappa}((z, w) & \left.,\left(z^{\prime}, w^{\prime}\right)\right) \\
= & \left(J_{1}\left(\left(\exp \overline{\left(z^{\prime}, w^{\prime}\right)}\right)^{-1},(z, w)\right)^{-1}, 0, J_{2}\left(\left(\exp \overline{\left(z^{\prime}, w^{\prime}\right)}\right)^{-1},(z, w)\right)\right)^{-1} \\
= & \left(J_{1}\left(\left(\exp \overline{\left(z^{\prime}, w^{\prime}\right)}\right)^{-1},(z, w)\right)^{-1}, 0,-J_{2}\left(\left(\exp \overline{\left(z^{\prime}, w^{\prime}\right)}\right)^{-1},(z, w)\right)\right) .
\end{aligned}
$$

Using (4.4), we obtain $\left(\left(\exp \overline{\left(z^{\prime}, w^{\prime}\right)}\right)^{-1}=\left(\exp \bar{z}^{\prime}, \bar{w}^{\prime}, 0\right)^{-1}=\left(\left(\exp \bar{z}^{\prime}\right)^{-1},-\bar{w}^{\prime}, 0\right)\right.$, and hence we see that

$$
J_{1}\left(\left(\exp \overline{\left(z^{\prime}, w^{\prime}\right)}\right)^{-1},(z, w)\right)^{-1}=J\left(\left(\exp \bar{z}^{\prime}\right)^{-1}, z\right)^{-1}=\kappa\left(z, z^{\prime}\right)
$$


where $J$ and $\kappa$ are the canonical automorphy factor and the canonical kernel function for the group $G$ given in (3.1) and (3.3), respectively. Thus we obtain

$$
\tilde{\kappa}\left((z, w),\left(z^{\prime}, w^{\prime}\right)\right)=\left(\kappa\left(z, z^{\prime}\right), 0, \kappa_{2}\left((z, w),\left(z^{\prime}, w^{\prime}\right)\right)\right),
$$

where

$$
\begin{aligned}
\kappa_{2}\left((z, w),\left(z^{\prime}, w^{\prime}\right)\right)= & -J_{2}\left(\left(\exp \overline{\left(z^{\prime}, w^{\prime}\right)}\right)^{-1},(z, w)\right) \\
= & \beta\left(\bar{w}^{\prime}, \rho\left(\kappa_{1}\left(z, z^{\prime}\right)\right)^{-1} \tau(z) \bar{w}^{\prime}\right) / 2 \\
& +\beta\left(\bar{w}^{\prime}, \rho\left(\kappa_{1}\left(z, z^{\prime}\right)\right)^{-1} w\right) \\
& +\beta\left(\overline{\tau\left(z^{\prime}\right)} w, \rho\left(\kappa_{1}\left(z, z^{\prime}\right)\right)^{-1} w\right) / 2 .
\end{aligned}
$$

We set

$$
\mathfrak{K}\left((z, w),\left(z^{\prime}, w^{\prime}\right)\right)=\mathbf{e}\left[\kappa_{2}\left((z, w),\left(z^{\prime}, w^{\prime}\right)\right)\right]
$$

for $(z, w),\left(z^{\prime}, w^{\prime}\right) \in \widetilde{\mathscr{D}}$.

PROPOSITION 6.1. Let $\mathscr{J}=\mathbf{e}\left[J_{2}\right]$ be as in (4.17). Then we have

$$
\mathfrak{K}\left(\left(z^{\prime}, w^{\prime}\right),(z, w)\right)=\overline{\mathfrak{K}\left((z, w),\left(z^{\prime}, w^{\prime}\right)\right)},
$$

for all $(z, w),\left(z^{\prime}, w^{\prime}\right) \in \widetilde{\mathscr{D}}$ and $\tilde{g} \in \widetilde{G}$.

ProOF. Let $(z, w),\left(z^{\prime}, w^{\prime}\right) \in \widetilde{\mathscr{D}}$ and $\tilde{g} \in \widetilde{G}$. Then by Lemma 4.1 the canonical kernel function $\tilde{\kappa}(\cdot, \cdot)$ satisfies the relations

$$
\begin{aligned}
\tilde{\kappa}\left(\left(z^{\prime}, w^{\prime}\right),(z, w)\right)= & \overline{\bar{\kappa}\left((z, w),\left(z^{\prime}, w^{\prime}\right)\right)^{-1}} \\
= & \left.\left(\overline{\kappa\left(z, z^{\prime}\right)}\right)^{-1}, 0,-\overline{\kappa_{2}\left((z, w),\left(z^{\prime}, w^{\prime}\right)\right)}\right), \\
\tilde{\kappa}\left(\tilde{g}(z, w), \tilde{g}\left(z^{\prime}, w^{\prime}\right)\right)= & \tilde{J}(\tilde{g},(z, w)) \tilde{\kappa}\left((z, w),\left(z^{\prime}, w^{\prime}\right)\right) \overline{J\left(\tilde{g},\left(z^{\prime}, w^{\prime}\right)\right)^{-1}} \\
= & \left(J(g, z), 0, J_{2}(\tilde{g},(z, w))\right)\left(\kappa\left(z, z^{\prime}\right), 0, \kappa_{2}\left((z, w),\left(z^{\prime}, w^{\prime}\right)\right)\right) \\
& \left.\times\left(\overline{J\left(g, z^{\prime}\right)}\right)^{-1}, 0,-\overline{J_{2}\left(\tilde{g},\left(z^{\prime}, w^{\prime}\right)\right)}\right) \\
= & \left(\kappa_{1}^{\prime}, 0, \kappa_{2}^{\prime}\right),
\end{aligned}
$$

where

$$
\begin{aligned}
& \kappa_{1}^{\prime}=J(g, z) \kappa\left(z, z^{\prime}\right) \overline{J\left(g, z^{\prime}\right)}{ }^{-1}, \\
& \kappa_{2}^{\prime}=J_{2}(\tilde{g},(z, w))+\kappa_{2}\left((z, w),\left(z^{\prime}, w^{\prime}\right)\right)-\overline{J_{2}\left(\tilde{g},\left(z^{\prime}, w^{\prime}\right)\right)} .
\end{aligned}
$$

In particular, we obtain

$$
\begin{aligned}
\kappa_{2}\left(\left(z^{\prime}, w^{\prime}\right),(z, w)\right) & =-\overline{\kappa_{2}\left((z, w),\left(z^{\prime}, w^{\prime}\right)\right)} \\
\kappa_{2}\left(\tilde{g}(z, w), \tilde{g}\left(z^{\prime}, w^{\prime}\right)\right) & =J_{2}(\tilde{g},(z, w))+\kappa_{2}\left((z, w),\left(z^{\prime}, w^{\prime}\right)\right)-\overline{J_{2}\left(\tilde{g},\left(z^{\prime}, w^{\prime}\right)\right)} .
\end{aligned}
$$


Thus it follows that

$$
\begin{aligned}
\mathfrak{K}\left(\left(z^{\prime}, w^{\prime}\right),(z, w)\right) & =\mathbf{e}\left[-\overline{\kappa_{2}\left((z, w),\left(z^{\prime}, w^{\prime}\right)\right)}\right]=\overline{\mathfrak{K}\left((z, w),\left(z^{\prime}, w^{\prime}\right)\right)}, \\
\mathfrak{K}\left(\tilde{g}(z, w), \tilde{g}\left(z^{\prime}, w^{\prime}\right)\right) & =\mathbf{e}\left[J_{2}(\tilde{g},(z, w))\right] \mathbf{e}\left[\kappa_{2}\left((z, w),\left(z^{\prime}, w^{\prime}\right)\right)\right] \mathbf{e}\left[-\overline{J_{2}\left(\tilde{g},\left(z^{\prime}, w^{\prime}\right)\right)}\right] \\
& =\mathscr{J}(\tilde{g},(z, w)) \mathfrak{K}\left((z, w),\left(z^{\prime}, w^{\prime}\right)\right) \overline{\mathscr{J}\left(\tilde{g},\left(z^{\prime}, w^{\prime}\right)\right)}
\end{aligned}
$$

hence the proof of the proposition is complete.

LEMMA 6.2. Let $\mathfrak{L}\left((z, w),\left(z^{\prime}, w^{\prime}\right)\right)$ be a $\mathbb{C}$-valued function on $\widetilde{\mathscr{D}} \times \widetilde{\mathscr{D}}$ that is holomorphic in $(z, w)$ and satisfies (6.3) and (6.4). Then $\mathfrak{L}$ is a constant multiple of $\mathfrak{K}$.

PROOF. For $\tilde{z}=(z, w), \tilde{z}^{\prime}=\left(z^{\prime}, w^{\prime}\right) \in \widetilde{\mathscr{D}}$ we set $\eta\left(\tilde{z}, \tilde{z}^{\prime}\right)=\mathfrak{L}\left(\tilde{z}, \tilde{z}^{\prime}\right) \mathfrak{K}\left(\tilde{z}, \tilde{z}^{\prime}\right)^{-1}$. Then, using (6.4), we obtain $\eta\left(\tilde{g} \tilde{z}, \tilde{g} \tilde{z}^{\prime}\right)=\eta\left(\tilde{z}, \tilde{z}^{\prime}\right)$ for all $\tilde{g} \in \widetilde{G}$. Thus, if $\tilde{z}_{0} \in \widetilde{\mathscr{D}}$ is a base point, then we have $\eta\left(\tilde{g} \tilde{z}_{0}, \tilde{z}_{0}\right)=\eta\left(\tilde{z}_{0}, \tilde{g}^{-1} \tilde{z}_{0}\right)$ for all $\tilde{g} \in \widetilde{G}$. Since $\eta\left(\tilde{z}, \tilde{z}^{\prime}\right)$ is holomorphic in $\tilde{z}$, by (6.3) it is antiholomorphic in $\tilde{z}^{\prime}$. Therefore, using the fact that $\widetilde{G}$ acts on $\widetilde{\mathscr{D}}$ transitively, we see that $\eta\left(\tilde{g} \tilde{z}_{0}, \tilde{z}_{0}\right)=\eta\left(\tilde{z}_{0}, \tilde{g}^{-1} \tilde{z}_{0}\right)=\eta\left(\tilde{z}_{0}, \tilde{z}_{0}\right)$ for all $\tilde{g} \in \widetilde{G}$. Thus, if $\tilde{z}, \tilde{z}^{\prime} \in \widetilde{\mathscr{D}}$ with $\tilde{z}^{\prime}=\tilde{g}^{\prime} \tilde{z}_{0}$, we obtain $\eta\left(\tilde{z}, \tilde{z}^{\prime}\right)=\eta\left(\left(\tilde{g}^{\prime}\right)^{-1} \tilde{z}, \tilde{z}_{0}\right)=\eta\left(\tilde{z}_{0}, \tilde{z}_{0}\right)$; hence it follows that $\mathfrak{k}\left(\tilde{z}, \tilde{z}^{\prime}\right)=C \mathfrak{L}\left(\tilde{z}, \tilde{z}^{\prime}\right)$ with $C=\eta\left(\tilde{z}_{0}, \tilde{z}_{0}\right)$.

Given elements $z \in \mathscr{D}$ and $w, w^{\prime} \in V_{+}$, we set

$$
\mathfrak{K}_{z}\left(w, w^{\prime}\right)=\mathscr{K}\left((z, w),\left(z, w^{\prime}\right)\right) .
$$

For each $z \in \mathscr{D}$ we denote by $\mathscr{F}_{z}$ the space of holomorphic functions $\phi$ on $V_{+}$such that

$$
\|\phi\|_{z}^{2}=\int_{V_{+}}|\phi(w)|^{2} \mathfrak{K}_{z}(w, w)^{-1} d_{z} w<\infty,
$$

where $d_{z} w=\operatorname{det}(\operatorname{Im} \tau(z))^{-1} d w$. Thus $\mathscr{F}_{z}$ together with the inner product

$$
\langle\phi, \psi\rangle_{z}=\int_{V_{+}} \phi(w) \overline{\psi(w)} \mathfrak{K}_{z}(w, w)^{-1} d_{z} w
$$

is a Hilbert space.

For $\tilde{g}=(g, v, t) \in \widetilde{G}$ and $\phi \in \mathscr{F}_{g z}$, we set

$$
\left(T^{g z}\left(\tilde{g}^{-1}\right) \phi\right)(w)=\mathscr{J}(\tilde{g},(z, w))^{-1} \phi\left(\operatorname{pr}_{2}((\tilde{g}(z, w)))\right.
$$

for all $(z, w) \in \mathscr{D} \times V_{+}$, where $\mathrm{pr}_{2}: \mathscr{D} \times V_{+} \rightarrow V_{+}$is the natural projection map onto $V_{+}$; hence we have

$$
\begin{aligned}
\operatorname{pr}_{2}((\tilde{g}(z, w))) & =\operatorname{pr}_{2}\left(g z, v_{g z}+J_{+}^{S}(\rho(g), \tau(z)) w\right) \\
& =v_{g z}+J_{+}^{S}(\rho(g), \tau(z)) w .
\end{aligned}
$$


LEMMA 6.3. For $\tilde{g}=(g, v, t) \in \widetilde{G}$ and $\phi \in \mathscr{F}_{g z}$, we have $T^{g z}\left(\tilde{g}^{-1}\right) \phi \in \mathscr{F}_{z}$ and $\left\|T^{g z}\left(\tilde{g}^{-1}\right) \phi\right\|_{z}=\|\phi\|_{g z}$ for all $z \in \mathscr{D}$.

ProOF. Let $z \in \mathscr{D}, \tilde{g}=(g, v, t) \in \widetilde{G}$ and $\phi \in \mathscr{F}_{g(z)}$. Then we have

$$
\begin{aligned}
\left\|T^{g z}\left(\tilde{g}^{-1}\right) \phi\right\|_{z}^{2} & =\int_{V_{+}}\left|T^{g z}\left(\tilde{g}^{-1}\right) \phi(w)\right|^{2} \Re_{z}(w, w)^{-1} d_{z} w \\
& =\int_{V_{+}}\left|\mathscr{J}(\tilde{g},(z, w))^{-1} \phi\left(v_{g z}+J_{+}^{S}(\rho(g), \tau(z)) w\right)\right|^{2} \Re_{z}(w, w)^{-1} d_{z} w .
\end{aligned}
$$

However, by Proposition 6.1, we have

$$
\begin{aligned}
\mathfrak{K}_{g z}\left(v_{g z}+J_{+}^{S}(\rho(g), \tau(z)) w, v_{g z}+J_{+}^{S}(\rho(g), \tau(z)) w\right) \\
\quad=\mathscr{J}(\tilde{g},(z, w)) \mathfrak{K}_{z}(w, w) \frac{\mathscr{J}(\tilde{g},(z, w))}{=}=|\mathscr{J}(\tilde{g},(z, w))|^{2} \mathfrak{K}_{z}(w, w) .
\end{aligned}
$$

Furthermore, we have

$$
\begin{aligned}
d_{g z}\left(v_{g z}\right. & \left.+J_{+}^{S}(\rho(g), \tau(z)) w\right) \\
& =\operatorname{det}(\operatorname{Im} \tau(g z))^{-1} d\left(v_{g z}+J_{+}^{S}(\rho(g), \tau(z)) w\right) \\
& =\left|J_{+}^{\prime}(\rho(g), \tau(z))\right|^{2} \operatorname{det}(\operatorname{Im} \tau(z))^{-1} d\left(v_{g z}+J_{+}^{S}(\rho(g), \tau(z)) w\right),
\end{aligned}
$$

where $J_{+}^{\prime}$ is the restriction of the canonical automorphy factor of $\operatorname{Sp}(V, \beta)$ given in (3.5) to $V_{+}$. However, we have $d\left(v_{g z}+J_{+}^{S}(\rho(g), \tau(z)) w\right)=\left|J_{+}^{S}(\rho(g), \tau(z))\right|^{-2}$, which implies that $d_{g z}\left(v_{g z}+J_{+}^{S}(\rho(g), \tau(z)) w\right)=d_{z} w$. Hence we have

$$
\begin{aligned}
\left\|T^{g z}\left(\tilde{g}^{-1}\right) \phi\right\|_{z}^{2}= & \int_{v_{+}}\left|\phi\left(v_{g z}+J_{+}^{S}(\rho(g), \tau(z)) w\right)\right|^{2} \\
& \times \Re_{g z}\left(v_{g z}+J_{+}^{S}(\rho(g), \tau(z)) w, v_{g z}+J_{+}^{S}(\rho(g), \tau(z)) w\right)^{-1} \\
& \times d_{g z}\left(v_{g z}+J_{+}^{S}(\rho(g), \tau(z)) w\right) \\
= & \int_{V_{+}}|\phi(w)|^{2} \Re_{g z}(w, w) d_{g z} w=\|\phi\|_{g z}^{2},
\end{aligned}
$$

and therefore the lemma follows.

By Lemma 6.3 we see that $T^{g z}\left(\tilde{g}^{-1}\right)$ is an isometry of $\mathscr{F}_{g z}$ into $\mathscr{F}_{z}$, and therefore it follows that $T^{z}(\tilde{g})$ is an isometry of $\mathscr{F}_{z}$ into $\mathscr{F}_{g z}$, and for $\phi \in \mathscr{F}_{z}$ we have

$$
\left(T^{z}(\tilde{g}) \phi\right)(w)=\left(T^{g^{-1}(g z)}(\tilde{g}) \phi\right)(w)=\mathscr{J}\left(\tilde{g}^{-1},(g z, w)\right)^{-1} \phi\left(\operatorname{pr}_{2}\left(\tilde{g}^{-1}(g z, w)\right)\right)
$$
for all $\tilde{z} \in \mathscr{D}$ and $w \in V_{+}$.

PROPOSITION 6.4. For $\tilde{g}=(g, v, t), \tilde{g}^{\prime}=\left(g^{\prime}, v^{\prime}, t^{\prime}\right) \in \widetilde{G}$ and $\phi \in \mathscr{F}_{g(z)}$, we have $T^{g^{\prime} z}(\tilde{g}) \circ T^{z}\left(\tilde{g}^{\prime}\right)=T^{z}\left(\tilde{g} \tilde{g}^{\prime}\right)$ for all $z \in \mathscr{D}$. 
PROOF. Let $\tilde{g}=(g, v, t), \tilde{g}^{\prime}=\left(g^{\prime}, v^{\prime}, t^{\prime}\right) \in \widetilde{G},(z, w) \in \widetilde{\mathscr{D}}=\mathscr{D} \times V_{+}$and $\phi \in \mathscr{F}_{g(z)}$. Then from (6.8) we obtain

$$
\left(T^{z}\left(\tilde{g}^{\prime}\right) \phi\right)(w)=\mathscr{J}\left(\tilde{g}^{\prime-1},\left(g^{\prime} z, w\right)\right)^{-1} \phi\left(\operatorname{pr}_{2}\left(\tilde{g}^{\prime-1}\left(g^{\prime} z, w\right)\right)\right) .
$$

Applying (6.8) once again, we see that

$$
\begin{aligned}
\left(T^{g^{\prime} z}(\tilde{g})\right. & \left.\circ T^{2}\left(\tilde{g}^{\prime}\right)(\phi)\right)(w) \\
= & \mathscr{J}\left(\tilde{g}^{-1},\left(g g^{\prime} z, w\right)\right)^{-1} \mathscr{J}\left(\tilde{g}^{\prime-1},\left(g^{\prime} z, \operatorname{pr}_{2}\left(\tilde{g}^{-1}\left(g g^{\prime} z, w\right)\right)\right)\right)^{-1} \\
& \times \phi\left(\operatorname{pr}_{2}\left(\tilde{g}^{\prime-1}\left(g^{\prime} z, \operatorname{pr}_{2}\left(\tilde{g}^{-1}\left(g g^{\prime} z, w\right)\right)\right)\right)\right) .
\end{aligned}
$$

On the other hand, we have

$$
\begin{aligned}
\left(T^{z}\left(\tilde{g} \tilde{g}^{\prime}\right)(\phi)\right)(w)= & \mathscr{J}\left(\left(\tilde{g} \tilde{g}^{\prime}\right)^{-1},\left(g g^{\prime} z, w\right)\right) \phi\left(\operatorname{pr}_{2}\left(\left(\tilde{g} \tilde{g}^{\prime}\right)^{-1}\left(g g^{\prime} z, w\right)\right)\right) \\
= & \mathscr{J}\left(\tilde{g}^{\prime-1}, \tilde{g}^{-1}\left(g g^{\prime} z, w\right)\right) \mathscr{J}\left(\tilde{g}^{-1},\left(g g^{\prime} z, w\right)\right) \\
& \times \phi\left(\operatorname{pr}_{2}\left(\left(\tilde{g} \tilde{g}^{\prime}\right)^{-1}\left(g g^{\prime} z, w\right)\right)\right) .
\end{aligned}
$$

Since we have

$$
\begin{gathered}
\tilde{g}^{-1}\left(g g^{\prime} z, w\right)=\left(g^{-1} g g^{\prime} z, \operatorname{pr}_{2}\left(\tilde{g}^{-1}\left(g g^{\prime} z, w\right)\right)\right)=\left(g^{\prime} z, \operatorname{pr}_{2}\left(\tilde{g}^{-1}\left(g g^{\prime} z, w\right)\right)\right), \\
\operatorname{pr}_{2}\left(\tilde{g}^{\prime-1}\left(g^{\prime} z, \operatorname{pr}_{2}\left(\tilde{g}^{-1}\left(g g^{\prime} z, w\right)\right)\right)\right)=\operatorname{pr}_{2}\left(\left(\tilde{g}^{\prime-1} \tilde{g}^{-1}\left(g g^{\prime} z, w\right)\right)\right. \\
=\operatorname{pr}_{2}\left(\left(\tilde{g} \tilde{g}^{\prime}\right)^{-1}\left(g g^{\prime} z, w\right)\right),
\end{gathered}
$$

it follows that $\left(T^{g^{\prime} z}(\tilde{g}) \circ T^{z}\left(\tilde{g}^{\prime}\right)(\phi)\right)(w)=\left(T^{z}\left(\tilde{g} \tilde{g}^{\prime}\right)(\phi)\right)(w)$, and therefore the proposition follows.

Now we consider the subgroup $\{1\} \times V_{+} \times \mathbb{R}$ of $\widetilde{G}$. We shall identify this subgroup with $\widetilde{V}=V_{+} \times \mathbb{R}$. Then $\widetilde{V}$ is in fact a Heisenberg group because the restriction of the multiplication operation on $\widetilde{G}$ given by (4.1) to $\widetilde{V}$ gives us the usual multiplication operation on a Heisenberg group. For $\tilde{u}=(u, t) \in \widetilde{V} \subset \widetilde{G}$ and $w \in V_{+}$we set $\tilde{u} w=\operatorname{pr}_{2}((\tilde{u}(z, w))$. Then, using (4.13), we obtain

$$
\tilde{u} w=\operatorname{pr}_{2}\left(((1, u, t)(z, w))=u_{1 z}+J_{+}^{S}(\rho(1), \tau(z)) w=u_{z}+w .\right.
$$

Thus for $\tilde{g}=\tilde{u}$ the formula (6.8) reduces to

$$
\left(T^{2}\left(\tilde{u}^{-1}\right) \phi\right)(w)=\mathscr{J}(\tilde{u},(z, w))^{-1} \phi(\tilde{u} w)
$$

for $\phi \in \mathscr{F}_{z}, z \in \mathscr{D}$ and $w \in V_{+}$, and $T^{z}\left(\tilde{u}^{-1}\right)$ is an isometry of $\mathscr{F}_{z}$ into itself.

LEMMA 6.5. For fixed $z \in \mathscr{D}$ the function $\mathfrak{F}_{z}\left(w, w^{\prime}\right)$ is holomorphic in $w$, and we have

$$
\begin{aligned}
\mathfrak{K}_{z}\left(w^{\prime}, w\right) & =\overline{\mathfrak{K}_{z}\left(w, w^{\prime}\right)}, \\
\mathfrak{K}_{z}\left(\tilde{u} w, \tilde{u} w^{\prime}\right) & =\mathscr{J}(\tilde{u},(z, w)) \mathfrak{K}_{z}\left(w, w^{\prime}\right) \overline{\mathscr{J}\left(\tilde{u},\left(z, w^{\prime}\right)\right)}
\end{aligned}
$$

for all $w, w^{\prime} \in V_{+}$and $\tilde{u} \in \tilde{V}$. 
PROOF. Using (6.1), (6.2) and (6.5), we have

$$
\mathfrak{F}_{z}\left(w, w^{\prime}\right)=\mathfrak{K}\left((z, w),\left(z, w^{\prime}\right)\right)=\mathbf{e}\left[\kappa_{2}\left((z, w),\left(z, w^{\prime}\right)\right)\right] .
$$

for $w, w^{\prime} \in V_{+}$, where

$$
\begin{aligned}
\kappa_{2}\left((z, w),\left(z, w^{\prime}\right)\right)= & \beta\left(\bar{w}^{\prime}, \rho\left(\kappa_{1}(z, z)\right)^{-1} \tau(z) \bar{w}^{\prime}\right) / 2+\beta\left(\bar{w}^{\prime}, \rho\left(\kappa_{1}(z, z)\right)^{-1} w\right) \\
& +\beta\left(\overline{\tau(z)} w, \rho\left(\kappa_{1}(z, z)\right)^{-1} w\right) / 2 .
\end{aligned}
$$

Thus $\kappa_{2}$ is holomorphic in $w$, and therefore $\mathcal{f}_{2}\left(w, w^{\prime}\right)$ is holomorphic in $w$. Now (6.11) and (6.12) follows from the corresponding relations in Proposition 6.1.

LEMMA 6.6. Let $\Psi\left(w, w^{\prime}\right)$ is a function on $V_{+} \times V_{+}$that is holomorphic in $w$ satisfying the conditions

$$
\begin{aligned}
\Psi\left(w^{\prime}, w\right) & =\overline{\Psi\left(w, w^{\prime}\right)}, \\
\Psi\left(\tilde{\boldsymbol{u}} w, \tilde{\boldsymbol{u}} w^{\prime}\right) & =\mathscr{J}(\tilde{\boldsymbol{u}},(z, w)) \Psi\left(w, w^{\prime}\right) \overline{\mathscr{J}\left(\tilde{\boldsymbol{u}},\left(z, w^{\prime}\right)\right)}
\end{aligned}
$$

for all $w, w^{\prime} \in V_{+}$and $\tilde{u} \in \tilde{V}$. Then $\Psi$ is a constant multiple of $\mathfrak{K}_{z}$.

Proof. This follows from Lemma 6.2.

For fixed $z \in \mathscr{D}$ the map $\phi \mapsto \phi(w), \mathscr{F}_{z} \rightarrow \mathbb{C}$ is a continuous linear functional on $\mathscr{F}_{z}$, and therefore there exists an element $\xi_{w}^{z} \in \mathscr{F}_{z}$ such that

$$
\phi(w)=\int_{V_{+}} \overline{\xi_{w}^{z}\left(w^{\prime}\right)} \phi\left(w^{\prime}\right) \mathfrak{K}_{z}\left(w^{\prime}, w^{\prime}\right)^{-1} d_{z} w^{\prime}
$$

for all $\phi \in \mathscr{F}_{z}$.

Lemma 6.7. Given $z \in \mathscr{D}$, there is a nonzero constant $C$ such that

$$
\overline{\xi_{w}^{z}\left(w^{\prime}\right)}=C \mathfrak{F}_{z}\left(w, w^{\prime}\right)
$$

for all $w, w^{\prime} \in V_{+}$.

PROOF. For $z \in \mathscr{D}$ and $w, w^{\prime} \in V_{+}$we have

$$
\xi_{w^{\prime}}^{z}(w)=\int_{V_{+}} \overline{\xi_{w}^{z}(v)} \xi_{w^{\prime}}^{z}(v) \mathfrak{K}_{z}(v, v)^{-1} d_{z} v,
$$

which implies that

$$
\overline{\xi_{w^{\prime}}^{z}(w)}=\int_{V_{+}} \xi_{w}^{z}(v) \overline{\xi_{w^{\prime}}^{z}(v)} \mathcal{K}_{z}(v, v)^{-1} d_{z} v=\xi_{w}^{z}\left(w^{\prime}\right)
$$


Let $\tilde{u}=(u, t) \in \widetilde{V} \subset \widetilde{G}$ and $\phi \in \mathscr{F}_{z}$, so that we have

$$
\left(T^{z}\left(\tilde{u}^{-1}\right) \phi\right)(w)=\mathscr{J}(\tilde{u},(z, w))^{-1} \phi(\tilde{u} w) .
$$

Then we see that

$$
\begin{aligned}
\int_{V_{+}} \overline{\xi_{w}^{z}(v)} \mathscr{J}(\tilde{u},(z, v))^{-1} \phi(\tilde{u} v) \mathcal{K}_{z}(v, v)^{-1} d_{z} v \\
\quad=\int_{V_{+}} \overline{\xi_{w}^{z}(v)}\left(T^{z}\left(\tilde{u}^{-1}\right) \phi\right)(v) \mathcal{K}_{z}(v, v)^{-1} d_{z} v \\
\quad=\mathscr{J}(\tilde{u},(z, w))^{-1} \int_{V_{+}} \overline{\xi_{\tilde{u} w}^{z}(v)} \phi(v) \mathcal{K}_{z}(v, v)^{-1} d_{z} v \\
=\mathscr{J}(\tilde{u},(z, w))^{-1} \int_{V_{+}} \overline{\xi_{\tilde{u} w}^{z}(\tilde{u} v)} \phi(\tilde{u} v) \mathcal{F}_{z}(\tilde{u} v, \tilde{u} v)^{-1} d_{z} v
\end{aligned}
$$

Thus we have

$$
\begin{aligned}
\mathscr{J}(\tilde{u}, & (z, v))^{-1} \overline{\xi_{w}^{z}(v)} \mathcal{\xi}_{z}(v, v)^{-1} \\
& =\mathscr{J}(\tilde{u},(z, w))^{-1} \overline{\xi_{\tilde{u} w}^{z}(\tilde{u} v)} \xi_{z}(\tilde{u} v, \tilde{u} v)^{-1} \\
& =\mathscr{J}(\tilde{u},(z, w))^{-1} \overline{\xi_{\tilde{u} w}^{z}(\tilde{u} v)} \mathscr{J}(\tilde{u},(z, v))^{-1} \overline{\mathscr{J}(\tilde{u},(z, v))^{-1} \mathfrak{K}_{z}(v, v)^{-1}}
\end{aligned}
$$

for $v \in V_{+}$. Hence, replacing $v$ with $w^{\prime}$, we see that

$$
\overline{\xi_{\tilde{u} w}^{z}\left(\tilde{u} w^{\prime}\right)}=\mathscr{J}(\tilde{u},(z, w)) \overline{\xi_{w}^{z}\left(w^{\prime}\right) \mathscr{J}\left(\tilde{u},\left(z, w^{\prime}\right)\right)} .
$$

Now the lemma follows by applying Lemma 6.6 to the function $\left(w, w^{\prime}\right) \mapsto \overline{\xi_{w}^{z}\left(w^{\prime}\right)}$.

Given an element $\tilde{v}$ of the Heisenberg group $\tilde{V}=V \times \mathbb{R}$, by (6.10) we obtain the isometry $T^{z}(\tilde{v})$ of $\mathscr{F}_{z}$ into itself given by

$$
\left(T^{z}(\tilde{v}) \phi\right)(w)=\mathscr{J}\left(\tilde{v}^{-1},(z, w)\right)^{-1} \phi\left(\tilde{v}^{-1} w\right)
$$

for all $w \in V_{+}$. We now consider an operator on $\mathscr{F}_{z}$ associated to a function on $\widetilde{V}$. Let $\mathscr{L}(\widetilde{V})$ be the space of $\mathbb{C}$-valued continuous functions on $\widetilde{V}$ with compact support. For $F \in \mathscr{L}(\tilde{V})$, we denote by $T^{z}(F)$ the operator on $\mathscr{F}_{z}$ defined by

$$
T^{z}(F) \phi=\int_{\tilde{v}} F(\tilde{v})\left(T^{z}(\tilde{v}) \phi\right) d \tilde{v}
$$

for all $\phi \in \mathscr{F}_{z}$.

LEMMA 6.8. For $F \in \mathscr{L}(\tilde{V})$ and $\phi \in \mathscr{F}_{z}$ we have

$$
\left(T^{z}(F) \phi\right)(w)=\int_{V_{+}} k_{z}\left(w, w^{\prime}\right) \phi\left(w^{\prime}\right) \Re_{z}\left(w^{\prime}, w^{\prime}\right)^{-1} d_{z} w^{\prime}
$$

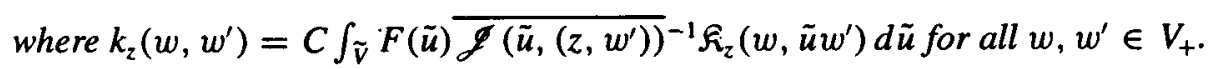


PROOF. Using (6.15) and Lemma 6.7, we have

$$
\left(T^{z}(F) \phi\right)(w)=\int_{V_{+}} \mathcal{K}_{z}\left(w, w^{\prime}\right)\left(T^{z}(F) \phi\right)\left(w^{\prime}\right) \mathcal{K}_{z}\left(w^{\prime}, w^{\prime}\right)^{-1} d_{z} w^{\prime}
$$

for $F \in \mathscr{L}(\tilde{V}), w \in V_{+}$and some constant $C$. On the other hand, for $\tilde{u} \in \tilde{V}$ we have

$$
\begin{aligned}
\left(T^{z}(\tilde{u}) \phi\right)(w) & =C \int_{V_{+}} \mathfrak{K}_{z}(w, v)\left(T^{z}(\tilde{u}) \phi\right)(v) \mathscr{K}_{z}(v, v)^{-1} d_{z} v \\
& =C \int_{V_{+}} \mathfrak{K}_{z}(w, v) \mathscr{J}\left(\tilde{u}^{-1},(z, v)\right)^{-1} \phi\left(\tilde{u}^{-1} v\right) \mathfrak{K}_{z}(v, v)^{-1} d_{z} v \\
& =C \int_{V_{+}} \mathfrak{K}_{z}\left(w, \tilde{u} w^{\prime}\right) \mathscr{J}\left(\tilde{u}^{-1}, \tilde{u}\left(z, w^{\prime}\right)\right)^{-1} \phi\left(w^{\prime}\right) \mathfrak{K}_{z}\left(\tilde{u} w^{\prime}, \tilde{u} w^{\prime}\right)^{-1} d_{z} w^{\prime} .
\end{aligned}
$$

Thus, using the relations

$$
\begin{gathered}
\mathfrak{K}_{z}\left(\tilde{u} w^{\prime}, \tilde{u} w^{\prime}\right)^{-1}=\overline{\mathscr{J}\left(\tilde{u},\left(z, w^{\prime}\right)\right)^{-1}} \mathfrak{K}_{z}\left(w^{\prime}, \tilde{u} w^{\prime}\right)^{-1} \mathscr{J}\left(\tilde{u},\left(z, w^{\prime}\right)\right)^{-1}, \\
\mathscr{J}\left(\tilde{u}^{-1}, \tilde{u}\left(z, w^{\prime}\right)\right) \mathscr{J}\left(\tilde{u},\left(z, w^{\prime}\right)\right)=\mathscr{J}\left(\tilde{u}^{-1} \tilde{u},\left(z, w^{\prime}\right)\right)=1,
\end{gathered}
$$

we obtain

$$
\left(T^{z}(\tilde{\boldsymbol{u}}) \phi\right)(w)=\int_{V_{+}} \mathfrak{K}_{z}\left(w, \tilde{\boldsymbol{u}} w^{\prime}\right) \overline{\mathscr{J}\left(\tilde{u},\left(z, w^{\prime}\right)\right)^{-1}} \mathfrak{K}_{z}\left(w^{\prime}, w^{\prime}\right)^{-1} \phi\left(w^{\prime}\right) d_{z} w^{\prime} .
$$

Hence we see that

$$
\begin{aligned}
\left(T^{z}(F) \phi\right)(w)= & \int_{V_{+}}\left(\int_{\tilde{v}} F(\tilde{u}) \overline{\left.\mathscr{J}\left(\tilde{u},\left(z, w^{\prime}\right)\right)^{-1} \mathfrak{K}_{z}\left(w, \tilde{u} w^{\prime}\right) d \tilde{u}\right)}\right. \\
& \times \phi\left(w^{\prime}\right) \mathfrak{K}_{z}\left(w^{\prime}, w^{\prime}\right)^{-1} d_{z} w^{\prime},
\end{aligned}
$$

and therefore the lemma follows.

THEOREM 6.9. Let $z$ be an element of the Hermitian symmetric domain $\mathscr{D}$ and let $\widetilde{V} \subset \widetilde{G}$ be the Heisenberg group associated to the real vector space $V$ described above. Then the map $\tilde{v} \mapsto T^{z}(\tilde{v})$ given by (6.16) is an irreducible unitary representation of $\widetilde{V}$ on the space $\mathscr{F}_{z}$.

Proof. By Proposition 6.4, for $\tilde{v}=(v, t), \tilde{v}^{\prime}=\left(v^{\prime}, t^{\prime}\right) \in \widetilde{V}$, we have

$$
T^{z}(\tilde{v}) \circ T^{z}\left(\tilde{v}^{\prime}\right)=T^{z}\left(\tilde{v} \tilde{v}^{\prime}\right)
$$

for all $z \in D$. Furthermore, using Lemma 6.3, we see that $\left\|T^{z}(\tilde{v}) \phi\right\|_{z}=\|\phi\|_{z}$ for all $z \in \mathscr{D}, \tilde{v} \in \widetilde{V}$ and $\phi \in \mathscr{F}_{z}$. Therefore the map $\tilde{v} \mapsto T^{z}(\tilde{v})$ determines a unitary 
representation of $\widetilde{V}$ on the space $\mathscr{F}_{z}$. It remains to show that $T^{z}$ is irreducible. Using Lemma 6.8, we see that the image of $\mathscr{F}_{z}$ under $T^{z}$ is dense in the ring of HilbertSchmidt operators on the space $\mathscr{L}_{2}^{2}\left(V_{+}\right)$of square-integrable functions on $V_{+}$with respect to the measure

$$
d \mu=\mathfrak{K}_{z}(w, w)^{-1} d_{z} w=\mathfrak{K}_{z}(w, w)^{-1} \operatorname{det}(\operatorname{Im} \tau(z))^{-1} d w
$$

for $w \in V_{+}$. This implies that the centralizer in Aut $\left(\mathscr{L}_{z}^{2}\left(V_{+}\right)\right)$of the image group of $\widetilde{V}$ under $T^{z}$ is the set $\mathbb{C}_{1}^{\times}$of complex numbers of modulus 1 . Indeed, each element $\lambda$ of the centralizer commutes with every $T^{z}(\tilde{u})$ for $\tilde{u} \in \tilde{V}$, and therefore with every $T^{z}(\phi)$ for $\phi \in \mathscr{F}_{z}$. By continuity $\lambda$ commutes with every element of the Hilbert space of Hilbert-Schmidt operators on $\mathscr{L}_{z}^{2}\left(V_{+}\right)$. Let $\psi_{1}, \psi_{2} \in \mathscr{L}_{z}^{2}\left(V_{+}\right)$, and let $\Xi$ be the Hilbert-Schmidt operator with kernel $k\left(w, w^{\prime}\right)=\psi_{1}(w) \overline{\psi_{2}\left(w^{\prime}\right)}$. Then we have $\Xi \lambda \psi=\lambda \Xi \psi$ for all $\psi \in L_{z}^{2}\left(V_{+}\right)$, which implies $\left\langle\psi, \psi_{2}\right\rangle \lambda \psi_{1}=\left\langle\lambda \psi, \psi_{2}\right\rangle \psi_{1}$. Since $\psi, \psi_{1}, \psi_{2}$ are arbitrary, it follows that $\lambda$ is a scalar; therefore the unitarity of $\lambda$ shows the claim that $\lambda \in \mathbb{C}_{1}^{x}$. Now let $\mathscr{F}_{z}^{1}$ be a $\widetilde{V}$ invariant subspace of $\mathscr{F}_{z}$ under $T^{z}$. Since $T^{z}$ is unitary, there is an invariant subspace $\mathscr{F}_{z}^{2}$ such that $\mathscr{F}_{z}=\mathscr{F}_{z}^{1} \oplus \mathscr{F}_{z}^{1}$. If $\Lambda$ is the scalr multiplication by $\lambda_{1} \in \mathbb{C}_{1}^{\times}$on $\mathscr{F}_{z}^{1}$ and $\lambda_{2} \in \mathbb{C}_{1}^{\times}$on $\mathscr{F}_{z}^{2}$ with $\lambda_{1} \neq t_{2}$, then $\Lambda$ belongs to the centralizer of the image group of $\widetilde{V}$ under $T^{z}$. Hence we have $\mathscr{F}_{z}^{2}=0$, and therefor $T^{z}$ is irreducible.

REMARK 6.10. If the Hermitian symmetric domain is the Siegel upper half space $\mathscr{H}_{n}$ and if $\rho$ and $\tau$ are identity maps, the representation $T^{z}$ given in Theorem 6.9 reduces to the usual Fock representation of the Heisenberg group $\widetilde{V}$ described in [21].

\section{Theta functions}

Let $(\rho, \tau)$ is the equivariant pair consisting of the homomorphism $\rho: G \rightarrow$ $\operatorname{Sp}(V, \beta)$ and the holomophic map $\tau: \mathscr{D} \rightarrow \mathscr{H}_{n}$ used for the construction of Kuga fiber varieties in Section 2. In this section we consider generalized theta functions on the Hermitian symmetric domain $\mathscr{D}$ which should reduce to usual theta functions on the Siegel upper half space $\mathscr{H}_{n}$ when $\rho$ and $\tau$ are identity maps. We obtain a transformation formula for such a theta function, and show that certain types of such theta functions genenate some eigenspaces associated to the Fock representations described in Section 6.

We shall use the same notations as in the previous sections. Thus $V$ is a real vector space of dimension $2 n$ whose complexification is of the form $V_{C}=V_{+}+V_{-}$, and the underlying real vector space of each of $V_{+}$and $V_{-}$is isomorphic to the real vector space $V$. Then there are $n$-dimensional subspaces $V_{1}$ and $V_{2}$ of $V$ and an element 
$\alpha \in \operatorname{Sp}(V, \beta)_{\mathrm{C}}$ such that $V=V_{1} \oplus V_{2}, \alpha\left(V_{1}\right)=V_{+}$and $\alpha\left(V_{2}\right)=V_{-}$. Let $L_{0}$ be a lattice in $V$ with $L=\alpha\left(L_{0}\right) \subset V_{\mathrm{C}}$ such that

$$
\beta(L, L) \subset \mathbb{Z}, \quad L=L \cap V_{+}+L \cap V_{-} .
$$

We set $L_{+}=L \cap V_{+}, L_{-}=L \cap V_{-}$and $V_{0}=\alpha(V) \subset V_{C}$. Thus each element $m \in V_{0}$ can be written in the form $m=m_{+}+m_{-} \in V_{0}$ with $m_{+}=V_{0} \cap V_{+}$and $m_{-}=V_{0} \cap V_{-}$.

DEFINITION 7.1. The theta function associated to $m \in V_{0}$ and the equivariant pair $(\rho, \tau)$ is the function $\theta_{m}: \mathscr{D} \times V_{+} \rightarrow \mathbb{C}$ given by

$$
\theta_{m}(z, w)=\sum_{l_{-} \in L_{-}} \mathbf{e}\left[\beta\left(l_{-}+m_{-}, \tau(z)\left(l_{-}+m_{-}\right)\right) / 2+\beta\left(l_{-}+m_{-}, w+m_{+}\right)\right]
$$

for all $(z, w) \in \mathscr{D} \times V_{+}$.

EXAMPLE 7.2. Let $S$ be an $r \times r$ real symmetric positive definite matrix, and let $\tau: \mathscr{H}_{k} \rightarrow \mathscr{H}_{k r}$ be the Eichler embedding (see for example [6, Section II.4]) given by $\tau(Z)=S \otimes Z$ for all $Z \in \mathscr{H}_{k}$, where $\mathscr{H}_{k}$ is regarded as the set of $k \times k$ complex symmetric matrices with positive definite imaginary part. Let $\rho: \operatorname{Sp}(k, \mathbb{R}) \rightarrow$ $\mathrm{Sp}(k r, \mathbb{R})$ be the homomorphism given by

$$
\rho\left(\begin{array}{ll}
A & B \\
C & D
\end{array}\right)=\left(\begin{array}{cc}
E \otimes A & S \otimes B \\
S^{-1} \otimes C & E \otimes D
\end{array}\right), \quad\left(\begin{array}{ll}
A & B \\
C & D
\end{array}\right) \in \mathrm{Sp}(k, \mathbb{R}),
$$

where $E$ is the $r \times r$ identity matrix. Then $(\rho, \tau)$ is an equivariant pair, and therefore (7.2) determines the associated theta function on $\mathscr{H}_{k} \times \mathbb{C}^{k r}$.

EXAMPLE 7.3. Let $\mathscr{H}^{k}$ be the product of $k$ copies of the Poincare upper half plane $\mathscr{H}$. We define the holomorphic map $\tau_{0}: \mathscr{H}^{h} \rightarrow \mathscr{H}_{k}$ and the homomorphism $\rho_{0}: \operatorname{Sp}(1, \mathbb{R})^{k} \rightarrow \operatorname{Sp}(k, \mathbb{R})$ as follows. Let $g=\left(g_{1}, \ldots, g_{k}\right)$ be an element of $\mathrm{Sp}(1, \mathbb{R})^{k}$ with $g_{i}=\left(\begin{array}{c}a_{i} b_{i} \\ c_{i}\end{array} d_{i}\right) \in \mathrm{Sp}(1, \mathbb{R})$ for $1 \leq i \leq k$, and let $z=\left(z_{1}, \ldots, z_{k}\right) \in \mathscr{H}^{k}$. Then we set

$$
\tau_{0}(z)=z^{*}, \quad \rho_{0}(g)=\left(\begin{array}{ll}
a^{*} & b^{*} \\
c^{*} & d^{*}
\end{array}\right),
$$

where $z^{*}=\operatorname{diag}\left(z_{1}, \ldots, z_{k}\right)$ is the $k \times k$ diagonal matrix and $a^{*}=\operatorname{diag}\left(a_{1}, \ldots, a_{k}\right)$, etc. Let $\Xi$ be an element of $\operatorname{Sp}(k, \mathbb{R})^{k}$, and set

$$
\tau(z)=\Xi \tau_{0}(z), \quad \rho(g)=\Xi \rho_{0}(g) \Xi^{-1}
$$

for $z \in \mathscr{H}^{k}$ and $g \in \mathrm{Sp}(k, \mathbb{R})^{k}$. Then $(\rho, \tau)$ is an equivariant pair, and (7.2) determines the associated theta function on $\mathscr{H}^{k}$. Such a function can be shown to be a Hilbert modular form under certain conditions if the results in [9] is used. 
LEMMA 7.4. Let $r=r_{+}+r_{-}$be an element of $L$ with $r_{+} \in L_{+}$and $r_{-} \in L_{-}$. Then we have

$$
\theta_{m}\left(z, w+r_{+}+\tau(z) r_{-}\right)=\mathbf{e}\left[-\beta\left(r_{-}, \tau(z) r_{-}\right) / 2-\beta\left(r_{-}, w+m_{+}\right)\right] \theta_{m+r}(z, w)
$$

for all $(z, w) \in \mathscr{D} \times V_{+}$.

Proof. Given $(z, w) \in \mathscr{D} \times V_{+}$, we have

$$
\begin{aligned}
\beta\left(l_{-}+\right. & \left.m_{-}, \tau(z)\left(l_{-}+m_{-}\right)\right) / 2+\beta\left(l_{-}+m_{-}, w+r_{+}+\tau(z) r_{-}+m_{+}\right) \\
= & \beta\left(l_{-}+m_{-}+r_{-}, \tau(z)\left(l_{-}+m_{-}+r_{-}\right)\right) / 2 \\
& -\beta\left(r_{-}, \tau(z)\left(l_{-}+m_{-}\right)\right) / 2-\beta\left(r_{-}, \tau(z) r_{-}\right) / 2-\beta\left(l_{-}+m_{-}, \tau(z) r_{-}\right) / 2 \\
& +\beta\left(l_{-}+m_{-}+r_{-}, w+r_{+}+m_{+}\right)+\beta\left(l_{-}+m_{-}, \tau(z) r_{-}\right) \\
& -\beta\left(r_{-}, w+r_{+}+m_{+}\right) .
\end{aligned}
$$

Since the matrix representation of $\tau(z): V_{C} \rightarrow V_{C}$ is of the form $\left(\begin{array}{c}0 \\ * \\ *\end{array}\right)$ relative to the decomposition $V_{\mathrm{C}}=V_{+} \oplus V_{-}$, we have $\tau(z)^{-1}=-{ }^{t} \tau(z)=-\tau(z)$; hence we obtain

$$
\beta\left(l_{-}+m_{-}, \tau(z) r_{-}\right)=\beta\left(\tau(z)^{-1}\left(l_{-}+m_{-}\right), r_{-}\right)=-\beta\left(\tau(z)\left(l_{-}+m_{-}\right), r_{-}\right) .
$$

Thus we see that

$$
\begin{aligned}
\beta\left(l_{-}+\right. & \left.m_{-}, \tau(z)\left(l_{-}+m_{-}\right)\right) / 2+\beta\left(l_{-}+m_{-}, w+r_{+} \tau(z) r_{-}+m_{+}\right) \\
= & \beta\left(l_{-}+m_{-}+r_{-}, \tau(z)\left(l_{-}+m_{-}+r_{-}\right)\right) / 2+\beta\left(l_{-}+m_{-}+r_{-}, w+r_{+}+m_{+}\right) \\
& -\beta\left(r_{-}, \tau(z) r_{-}\right) / 2-\beta\left(r_{-}, w+r_{+}+m_{+}\right),
\end{aligned}
$$

and therefore the lemma follows.

Given an element $l=l_{+}+l_{-} \in L$ with $l_{+} \in L_{+}$and $l_{-} \in L_{-}$, we set

$$
\psi_{m}(l)=\mathbf{e}\left[\beta\left(l_{+}, l_{-}\right) / 2+\beta\left(l_{-}, m_{+}\right)+\beta\left(m_{-}, l_{+}\right)\right] .
$$

Then $\psi_{m}$ is a quasi-character of $L$ in the sense that the map

$$
l \mapsto \psi_{m}(l) \mathbf{e}\left[\beta\left(l_{+}, l_{-}\right) / 2\right]
$$

is a character of $L$. We also set $l_{z}=l_{+}-\tau(z) l_{-} \in V_{+}$for $z \in \mathscr{D}$ as in Section 2 .

THEOREM 7.5. Let $\mathscr{J}: \widetilde{G} \times \widetilde{\mathscr{D}} \rightarrow \mathbb{C}$ be the automorphy factor given by (4.17). Then the theta function $\theta_{m}$ satisfies the relation

$$
\theta_{m}\left(z, w+l_{z}\right)=\psi_{m}(l) \mathscr{J}((1, l, 0),(z, w)) \theta_{m}(z, w)
$$

for all $(z, w) \in \widetilde{\mathscr{D}}=\mathscr{D} \times V_{+}$and $l \in L \subset V_{0}$. 
PROOF. Applying Lemma 7.4 for $(z, w) \in \mathscr{D} \times V_{+}$and $r=l_{+}-l_{-}$with $r_{+}=l_{+} \epsilon$ $L_{+}$and $r_{-}=-l_{-} \in L_{-}$, we have

$$
\theta_{m}\left(z, w+l_{z}\right)=\mathbf{e}\left[-\beta\left(l_{-}, \tau(z) l_{-}\right) / 2+\beta\left(l_{-}, w+m_{+}\right)\right] \theta_{m+r}(z, w) .
$$

However, for $m+r=\left(m_{+}+l_{+}\right)+\left(m_{-}-l_{-}\right)$, we have

$$
\begin{aligned}
\theta_{m+r}(z, w)= & \sum_{k_{-} \in L_{-}} \mathbf{e}\left[\beta\left(k_{-}+m_{-}-l_{-}, \tau(z)\left(k_{-}+m_{-}-l_{-}\right)\right) / 2\right. \\
& \left.+\beta\left(k_{-}+m_{-}-l_{-}, w+m_{+}+l_{+}\right)\right] \\
= & \sum_{k_{-} \in L_{-}} \mathbf{e}\left[\beta\left(k_{-}+m_{-}, \tau(z)\left(k_{-}+m_{-}\right)\right) / 2\right. \\
& \left.+\beta\left(k_{-}+m_{-}, w+m_{+}+l_{+}\right)\right] \\
= & \sum_{k_{-} \in L_{-}} \mathbf{e}\left[\beta\left(k_{-}+m_{-}, \tau(z)\left(k_{-}+m_{-}\right)\right) / 2\right. \\
& \left.+\beta\left(k_{-}+m_{-}, w+m_{+}\right)+\beta\left(k_{-}, l_{+}\right)+\beta\left(m_{-}, l_{+}\right)\right] \\
= & \mathbf{e}\left[\beta\left(m_{-}, l_{+}\right)\right] \theta_{m}(z, w),
\end{aligned}
$$

where we used the condition $\beta(L, L) \subset \mathbb{Z}$. Thus we obtain

$$
\theta_{m}\left(z, w+l_{z}\right)=\mathbf{e}\left[-\beta\left(l_{-}, \tau(z) l_{-}\right) / 2+\beta\left(l_{-}, w+m_{+}\right)+\beta\left(m_{-}, l_{+}\right)\right] \theta_{m}(z, w) .
$$

Since $\beta=0$ on $V_{+} \times V_{+}$and $V_{-} \times V_{-}$, we have

$$
\begin{aligned}
\mathscr{J}((1, l, 0),(z, w)) & =\mathbf{e}\left[\beta\left(l, l_{z}\right) / 2+\beta(l, w)\right]=\mathbf{e}\left[\beta\left(l_{-}, l_{z}\right) / 2+\beta\left(l_{-}, w\right)\right] \\
& =\mathbf{e}\left[\beta\left(l_{-}, l_{+}\right) / 2-\beta\left(l_{-}, \tau(z) l_{-}\right) / 2+\beta\left(l_{-}, w\right)\right] .
\end{aligned}
$$

Hence we see that

$$
\begin{aligned}
\psi(l) \mathcal{J}((1, l, 0),(z, w))= & \mathbf{e}\left[-\beta\left(l_{-}, \tau(z) l_{-}\right) / 2+\beta\left(l_{-}, w\right\}\right. \\
& \left.+\beta\left(l_{-}, m_{+}\right)+\beta\left(m_{-}, l_{+}\right)\right],
\end{aligned}
$$

and therefore the proof of the theorem is complete.

REMARK 7.6. If we set $\tilde{u}=(1, l, 0) \in \widetilde{G}$ and $\tilde{z}=(z, w) \in \widetilde{\mathscr{D}}$, then (7.4) can be written in the form

$$
\theta_{m}(\tilde{u} \tilde{z})=\mathscr{J}_{\psi}(\tilde{u}, \tilde{z}) \theta_{m}(\tilde{z}),
$$

where $\mathscr{I}_{\psi}:(\Gamma \times L \times\{0\}) \times\left(\mathscr{D} \times V_{+}\right) \rightarrow \mathbb{C}$ is the automorphy factor given by

$$
\mathscr{J}_{\psi}((\gamma, l, 0),(z, w))=\psi(l) \mathscr{J}((\gamma, l, 0),(z, w))
$$

for all $\gamma \in \Gamma, l \in L$ and $(z, w) \in \mathscr{D} \times V_{+}$. 
Given an element $z \in \mathscr{D}$ and a quasi-character $\psi$ of $L$, we denote by $V_{\psi}^{z}$ the complex vector space consisting of all functions $f: V_{+} \rightarrow \mathbb{C}$ satisfying the relation $T^{z}((1, l, 0)) f=\psi(l)^{-1} f$ for all $l \in L$.

PROPOSITION 7.7. Let $z \in \mathscr{D}$, and let $f$ be an element of $V_{\psi}^{z}$ for some quasicharacter $\psi$ of $L$. Then $T_{(\gamma, 0,0)}^{z} f$ is an element of $V_{\psi}^{\gamma z}$ for all $\gamma \in G$.

Proof. Let $l \in L, \gamma \in G$ and $z \in \mathscr{D}$. Then we have

$$
(1, \gamma l, 0)(\gamma, 0,0)=(\gamma, 0,0)(1, l, 0)=(\gamma, \gamma l, 0) \in \widetilde{G}=G \times V_{0} \times \mathbb{R} .
$$

Hence, using Proposition 6.4, we obtain

$$
T^{\gamma z}((\gamma, 0,0)) \circ T^{z}((1, l, 0))=T^{z}((\gamma, 0,0)) \circ T^{z}((1, l, 0)) .
$$

Thus for $f \in V_{\psi}^{z}$, we have

$$
\begin{aligned}
T^{\gamma z}((\gamma, 0,0))\left(T^{z}((1, l, 0)) f\right) & =T^{z}((\gamma, 0,0))\left(T^{z}((1, l, 0)) f\right) \\
& =\psi(l)^{-1}\left(T^{z}((\gamma, 0,0)) f\right),
\end{aligned}
$$

and therefore we see that $T^{z}((1, l, 0)) f \in V_{\psi}^{\gamma z}$.

Given $z \in \mathscr{D}$ and $m \in V_{0}$ we define the function $\theta_{m}^{z}: V_{+} \rightarrow \mathbb{C}$ by

$$
\theta_{m}^{z}(w)=\theta_{m}(z, w)
$$

for all $w \in V_{+}$.

Proposition 7.8. Let $z \in \mathscr{D}$, and set $\widehat{l}=(1, l, 0) \in \widetilde{G}$ for $l \in L$. Let $T^{z}(\widehat{l})$ be the associated operator on $\mathscr{F}_{z}$ given by (6.16), and let $\psi_{m}$ be the quasi-character associated to $m \in V_{0}$ in (7.3). Then the function $\theta_{m}^{z}$ is an element of $V_{\psi_{m}}^{z}$.

PROOF. Given $z \in \mathscr{D}, m \in V_{0}, \widehat{l}=(1, l, 0) \in \widetilde{G}$ and $w \in V_{\mathbf{C}}$, we have

$$
\left.\left(T^{z}(\widehat{l})\left(\theta_{m}^{z}\right)\right)(w)=\mathscr{J}\left(\widehat{l}^{-1},(z, w)\right)^{-1} \theta_{m}^{z}\left(\widehat{l}^{-1}(z, w)\right)_{w}\right) .
$$

However, we have

$$
\hat{l}^{-1}(z, w)=(1,-l, 0)(z, w)=\left(z, w-l_{z}\right) ;
$$

hence we obtain $\left.\widehat{l}^{-1}(z, w)\right)_{w}=w-l_{z}$. Thus we see that

$$
\begin{aligned}
\left(T^{z}(\widehat{l})\left(\theta_{m}^{z}\right)\right)(w) & =\mathscr{J}\left(\widehat{l}^{-1},(z, w)\right)^{-1} \theta_{m}^{z}\left(w-l_{z}\right) \\
& =\mathscr{J}\left(\widehat{l}^{-1},(z, w)\right)^{-1} \psi_{m}(-l) \mathscr{J}\left(\widehat{l}^{-1},(z, w)\right) \theta_{m}^{z}(w) \\
& =\psi_{m}(-l) \theta_{m}^{z}(w)=\psi_{m}(l)^{-1} \theta_{m}^{z}(w) .
\end{aligned}
$$

Thus the proposition follows. 
Let $L_{+}^{*}$ be the dual lattice of $L_{+}$relative to $\beta$, that is,

$$
L_{+}^{*}=\left\{v \in V_{0} \mid \beta\left(L_{+}, v\right) \subset \mathbb{Z}\right\}
$$

Then by (7.1), we have $L_{-} \subset L_{+}^{*}$. Now we state the main theorem in this section which extends a result of Satake [21, Section 3] to the case of Hermitian symmetric domains.

THEOREM 7.9. Let $\Omega$ be the complete set of representatives $L_{+}$modulo $L_{-}$. Then, for $z \in \mathscr{D}$ and $m \in V_{0}$, the set $\left\{\theta_{m+r}^{z} \mid r \in \Omega\right\}$ forms a basis of the complex vector space $V_{\psi_{m}}^{z}$.

PROOF. Since the set $\left\{\theta_{m+r}^{z} \mid r \in \Omega\right\}$ is obviously linearly independent over $\mathbb{C}$, it suffices to show that it spans the complex vector space $V_{\psi_{m}}^{z}$. Let $z \in \mathscr{D}, w \in V_{0}$ and $m=m_{+}+m_{-}$with $m_{+} \in V_{+}$and $m_{-} \in V_{-}$. Then for $l_{+} \in L_{+}$we have

$$
\mathscr{J}\left(\left(1, l_{+}, 0\right),(z, w)\right)=1, \quad \psi\left(l_{+}\right)=\mathbf{e}\left[\beta\left(m_{-}, l_{+}\right)\right] .
$$

Thus, for $f \in V_{\psi_{m}}^{z}$, the relation $T_{\left(1, l_{+}, 0\right)}^{z} f=\psi_{m}\left(l_{+}\right)^{-1} f(w)$ reduces to

$$
f\left(w-l_{+}\right)=\mathbf{e}\left[-\beta\left(m_{-}, l_{+}\right)\right] f(w) .
$$

Hence the function $f_{e}(w)=f(w) \mathbf{e}\left[-\beta\left(m_{-}, w\right)\right]$ satisfies the relation

$$
\begin{aligned}
f_{e}\left(w-l_{+}\right) & =f\left(w-l_{+}\right) \mathbf{e}\left[-\beta\left(m_{-}, w\right)+\beta\left(m_{-}, l_{+}\right)\right] \\
& =f(w) \mathbf{e}\left[-\beta\left(m_{-}, l_{+}\right)\right] \mathbf{e}\left[-\beta\left(m_{-}, w\right)\right] \mathbf{e}\left[\beta\left(m_{-}, l_{+}\right)\right]=f_{e}(w) .
\end{aligned}
$$

Therefore we obtain a Fourier expansion of $f_{e}(w)$ of the form

$$
f_{e}(w)=f(w) \mathbf{e}\left[-\beta\left(m_{-}, w\right)\right]=\sum_{r \in L_{+}^{*}} a(r) \mathbf{e}[\beta(r, w)],
$$

which implies that $f(w)=\sum_{r \in L_{+}^{*}} a(r) \mathbf{e}\left[\beta\left(r+m_{-}, w\right)\right]$. On the other hand, for $l_{-} \in L_{-}$, we have

$$
f\left(w-\tau(z) l_{-}\right)=\mathbf{e}\left[-\beta\left(l_{-}, \tau(z) l_{-}\right) / 2+\beta\left(l_{-}, w\right)+\beta\left(l_{-}, m_{+}\right)\right] f(w) .
$$

By comparing the coefficients of $\mathbf{e}\left[\beta\left(r+m_{-}, w\right)\right]$ in the Fourier series of both sides of the above equation, we see that

$$
a(r) \mathbf{e}\left[\beta\left(r+m_{-}, \tau(z) l_{-}\right)\right]=a\left(r-l_{-}\right) \mathbf{e}\left[-\beta\left(l_{-}, \tau(z) l_{-}\right) / 2+\beta\left(l_{-}, m_{+}\right)\right]
$$

hence we have

$$
a\left(r-l_{-}\right)=a(r) \mathbf{e}\left[\beta\left(r+m_{-}, \tau(z) l_{-}\right)+\beta\left(l_{-}, \tau(z) l_{-}\right) / 2-\beta\left(l_{-}, m_{+}\right)\right]
$$


for each $r \in L_{+}^{*}$. Using this relation, we obtain

$$
\begin{aligned}
f(w)= & \sum_{r \in \Omega} \sum_{l_{-} \in L_{-}} a\left(r-l_{-}\right) \mathbf{e}\left[\beta\left(r+m_{-}-l_{-}, w\right)\right] \\
= & \sum_{r \in \Omega} \sum_{l_{-} \in L_{-}} a(r) \mathbf{e}\left[\beta\left(r+m_{-}, \tau(z) l_{-}\right)+\beta\left(l_{-}, \tau(z) l_{-}\right) ; \dot{2}\right. \\
& \left.-\beta\left(l_{-}, m_{+}\right)+\beta\left(r+m_{-}-l_{-}, w\right)\right] .
\end{aligned}
$$

However, we have

$$
\begin{aligned}
& \beta\left(r+m_{-}, \tau(z) l_{-}\right)+\beta\left(l_{-}, \tau(z) l_{-}\right) / 2 \\
& \quad=\beta\left(r+m_{-}, \tau(z) l_{-}\right) / 2+\beta\left(l_{-}, \tau(z)\left(r+m_{-}\right)\right) / 2+\beta\left(l_{-}, \tau(z) l_{-}\right) / 2 \\
& \quad=\beta\left(r+m_{-}, \tau(z) l_{-}\right) / 2+\beta\left(l_{-}, \tau(z)\left(r+m_{-}+l_{-}\right)\right) / 2 \\
& \quad=\beta\left(r+m_{-}+l_{-}, \tau(z)\left(r+m_{-}+l_{-}\right)\right) / 2-\beta\left(r+m_{-}, \tau(z)\left(r+m_{-}\right)\right) / 2, \\
& -\beta\left(l_{-}, m_{+}\right)+\beta\left(r+m_{-}, w\right)=\beta\left(r+m_{-}-l_{-}, w+m_{+}\right)+\beta\left(r+m_{-}, m_{+}\right) .
\end{aligned}
$$

Thus we see that

$$
\begin{aligned}
f(w)= & \sum_{r \in \Omega} a(r) \mathbf{e}\left[\beta\left(r+m_{-}-l_{-}, \tau(z)\left(r+m_{-}\right)\right) / 2-\beta\left(r+m_{-}, m_{+}\right)\right] \\
& \times \sum_{l_{-} \in L_{-}} \mathbf{e}\left[\beta\left(r+m_{-}+l_{-}, \tau(z)\left(r+m_{-}+l_{-}\right)\right) / 2+\beta\left(r+m_{-}-l_{-}, w+m_{+}\right)\right] \\
= & \sum_{r \in \Omega} c(r, z) \theta_{m+r}^{z}(w),
\end{aligned}
$$

where $c(r, z)=a(r) \mathbf{e}\left[\beta\left(r+m_{-}, \tau(z)\left(r+m_{-}\right)\right) / 2-\beta\left(r+m_{-}, m_{+}\right)\right]$is a constant independent of $w$; hence the theorem follows.

\section{References}

[1] S. Abdulali, 'Conjugates of strongly equivariant maps', Pacific J. Math. 165 (1994), 207-216.

[2] S. Addington, 'Equivariant holomorphic maps of symmetric domains', Duke Math. J. 55 (1987), 65-88.

[3] R. Berndt and S. Böcherer, 'Jacobi forms and discrete series representations of the Jacobi group', Math. Z 204 (1990), 13-44.

[4] R. Borcherds, 'Automorphic forms on $O_{s+2.2}(\mathbf{R})$ and infinite products', Invent. Math. 120 (1995), 161-213.

[5] G. Faltings and C. L. Chai, Degeneration of abelian varieties (Springer, Berlin, 1990).

[6] E. Freitag, Singular modular forms and theta relations, Lecture Notes in Math. 1487 (Springer, Heidelberg, 1991). 
[7] E. Freitag and C. Hermann, 'Some modular varieties of low dimension', Adv. Math. 152 (2000), 203-287.

[8] B. B. Gordon, 'Algebraic cycles in families of abelian varieties over Hilbert-Blumenthal surfaces', J. Reine Angew. Math. 449 (1994), 149-171.

[9] W. Hammond, 'The modular groups of Hilbert and Siegel', in: Algebraic Groups and Discontinuous Subgroups (Proc. Sympos. Pure Math., Boulder, Colo., 1965) (Amer. Math. Soc., Providence, 1966) pp. 358-360.

[10] K. Hulek, C. Kahn and S. Weintraub, Moduli spaces of abelian surfaces: compactification, degenerations and theta functions (Walter de Gruyter, Berlin, 1993).

[11] J.-I. Igusa, Theta functions (Springer, Heidelberg, 1972).

[12] M. Kuga, Fiber varieties over a symmetric space whose fibers are abelian varieties I, II (Univ. of Chicago, Chicago, 1963/64).

[13] H. Lange and Ch. Birkenhake, Complex abelian varieties (Springer, Berlin, 1992).

[14] M. H. Lee, 'Conjugates of equivariant holomorphic maps of symmetric domains', Pacific J. Math. 149 (1991), 127-144.

[15] — 'Twisted torus bundles over arithmetic varieties', Proc. Amer. Math. Soc. 123 (1995), 2251-2259.

[16] _ 'Mixed automorphic vector bundles on Shimura varieties', Pacific J. Math. 173 (1996), 105-126.

[17] _ 'Generalized Jacobi forms and abelian schemes over arithmetic varieties', Collect. Math. 49 (1998), 121-131.

[18] _ - 'Jacobi forms on symmetric domains and torus bundles over abelian schemes', J. Lie Theory 11 (2001), 545-557.

[19] D. Mumford, Tata lectures on theta III (Birkhäuser, Boston, 1991).

[20] S. Murakami, Cohomology of vector-valued forms on symmetric spaces (Univ. of Chicago, Chicago, 1966).

[21] I. Satake, 'Fock representations and theta-functions', in: Advances in the Theory of Riemann Surfaces (Proc. Conf., Stony Brook, NY, 1969) Ann. of Math. Studies 66 (Princeton Univ. Press, 1971) pp. 393-405.

[22] - Algebraic structures of symmetric domains (Princeton Univ. Press, Princeton, 1980).

[23] M. Taylor, Noncommutative harmonic analysis (Amer. Math. Soc., Providence, 1986).

[24] C. Ziegler, 'Jacobi forms of higher degree', Abh. Math. Sem. Univ. Hamburg 59 (1989), 191-224.

\section{Department of Mathematics}

University of Northern Iowa

Cedar Falls, Iowa 50614

USA

e-mail: lee@math.uni.edu 